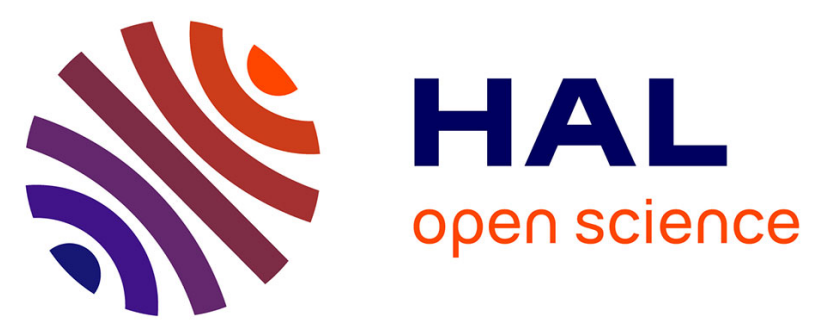

\title{
FFT-based micromechanical simulations of transformation plasticity. Comparison with a limit-analysis-based theory
}

Youssri El Majaty, Renald Brenner, Jean-Baptiste Leblond

\section{To cite this version:}

Youssri El Majaty, Renald Brenner, Jean-Baptiste Leblond. FFT-based micromechanical simulations of transformation plasticity. Comparison with a limit-analysis-based theory. European Journal of Mechanics - A/Solids, 2021, 86, pp.104152. 10.1016/j.euromechsol.2020.104152 . hal-03005756

\section{HAL Id: hal-03005756 https://hal.science/hal-03005756}

Submitted on 18 Nov 2020

HAL is a multi-disciplinary open access archive for the deposit and dissemination of scientific research documents, whether they are published or not. The documents may come from teaching and research institutions in France or abroad, or from public or private research centers.
L'archive ouverte pluridisciplinaire HAL, est destinée au dépôt et à la diffusion de documents scientifiques de niveau recherche, publiés ou non, émanant des établissements d'enseignement et de recherche français ou étrangers, des laboratoires publics ou privés. 


\title{
FFT-based micromechanical simulations of transformation plasticity. Comparison with a limit-analysis-based theory
}

\author{
Youssri EL MAJATY*, Renald BRENNER and Jean-Baptiste LEBLOND \\ Sorbonne Université, Centre National de la Recherche Scientifique, UMR 7190, Institut Jean Le Rond d’Alembert, F-75005 Paris, France
}

\section{ARTICLE INFO}

Keywords:

FFT-based simulations

Transformation plasticity

Greenwood-Johnson's mechanism

JMAK's model

\begin{abstract}
A B S TR ACT
This work addresses the numerical simulation of transformation plasticity by using a numerical scheme based on the fast Fourier transform (FFT). A two-phase material with isotropic thermoelastoplastic phases is considered. Together with prescribed transformation kinetics, this permits to describe the plasticity induced by the accommodation of the volume change accompanying the phase transformation (Greenwood-Johnson mechanism). We consider random distributions of $\alpha$-phase nuclei within a homogeneous $\gamma$-phase matrix, with an isotropic growth law of the nuclei. The numerical results are compared to a recently proposed limit-analysis-based theory (E1 Majaty et al., 2018), which permits in particular to account for a nonlinear dependence of the "transformation plastic strain" with the stress applied. A very good agreement between the FFT simulations and the theory is obtained, for uniaxial and multiaxial loadings, over a wide range of stresses applied.
\end{abstract}

\section{Introduction}

Transformation plasticity occurs during thermomechanical treatments of steels and metallic alloys, such as welding or quenching processes. This phenomenon induces residual stresses and distortions inside the materials, which modify their macroscopic mechanical behaviour; see, among others (Miyao et al., 1986; Fukumuto et al., 2001; Taleb et al., 2004). It is commonly accepted that transformation plasticity is due to two main mechanisms, a diffusive one (Greenwood and Johnson, 1965) and a displacive one (Magee and Paxton, 1966). The latter mechanism is not considered in the present study - see comments on this topic in El Majaty et al. (2018).

The origin of Greenwood and Johnson (1965)'s mechanism of transformation plasticity lies in the plastic accommodation of the austenite (softer phase) during the phase transformation. Indeed during cooling, the austenitic phase (face centered cubic structure) gives birth to a ferritic, bainitic or martensitic phase (body centered cubic or tetragonal structure) which has a larger specific volume. The volume difference between the two phases, equivalent to a heterogeneous eigenstrain field, generates internal stresses which cause local plastic deformations, even without any applied macroscopic stress. If present, such a stress takes advantage of this "pre-existing" microplasticity to deform the material more easily than in the absence of a transformation.

The first truly micromechanical modelling of this mechanism is due to Leblond et al. (1989), who considered a volume element made of a growing spherical ferritic, bainitic or martensitic nucleus ( $\alpha$-phase) surrounded by a concentric spherical austenitic shell ( $\gamma$-phase). Assuming a low applied macroscopic stress, this approach led to an expression of the overall transformation plastic strain rate depending linearly on the overall stress deviator.

\footnotetext{
*Corresponding author

@youssri@dalembert.fr ( EL MAJATY)

ORCID(s): $0000-0001-9686-7351$ (EL MAJATY)
}

Leblond et al. (1989)'s model was not, however, free of drawbacks and limitations. For instance, it predicted a physically unrealistic infinite value for the transformation plastic strain rate at the very start of the transformation. In addition, its derivation was based on the assumption of small stresses applied, so that it could not account for the well documented nonlinear increase of the transformation plastic strain rate with the stress applied when it becomes comparable to the yield stress of austenite (see for example Figures 3 to 6 in the experimental section of Greenwood and Johnson (1965)'s paper).

Extensions of this model have been proposed in various directions. The issue of the infinite initial value of the transformation plastic strain rate was dealt with, among others, by Taleb and Sidoroff (2003) and Weisz-Patrault (2017). Viscous effects were introduced by Vincent et al. (2003). Various mean-field models were used to refine the description of the mechanical behaviour of the mother-phase undergoing plastic deformations (see, for instance, Diani et al., 1995; Cherkaoui et al., 2000; Fischlschweiger et al., 2012, among others).

In connection with these investigations, numerical micromechanical simulations of transformation plasticity were performed by various authors, with different hypotheses concerning the processes of nucleation and growth of nuclei of daughter-phase. Most of these studies used the finite element method; see, among others, the works of Leblond et al. (1989); Ganghoffer et al. (1992); Barbe et al. (2007, 2008); Barbe and Quey (2011), which used von Mises's standard macroscopic model to describe plastic accommodation in the austenitic phase. However more recently another, FFTbased numerical scheme (Moulinec and Suquet, 1998) was used by Otsuka (2014); Otsuka et al. (2018) in conjunction with a more refined description of plasticity in the austenite based on a crystal plasticity model. ${ }^{1}$

However, in spite of these efforts, Leblond et al. (1989)'s

${ }^{1}$ The related, but distinct problem of TRIP/TWIP steels was also studied with the same numerical scheme by Wong et al. (2016). 
model predictions remain consistent with experimental observations only for macroscopic applied stresses less than approximately half of the yield stress of austenite. Thus, to tackle the range of high stresses close to the yield stress of austenite, the theory requires additional adjustable parameters.

To overcome the shortcomings of Leblond et al. (1989)'s model, the modelling of the Greenwood-Johnson mechanism was reconsidered by El Majaty et al. (2018) by making use of the Hill-Mandel theory of homogenization, combined with a limit-analysis kinematical approach. El Majaty et al. (2018)'s new model of transformation plasticity was compared, with globally satisfying results, to experiments performed on the A533 steel (Desalos, 1981; Coret et al., 2002, 2004).

The present study aims at investigating the relevance of El Majaty et al. (2018)'s new theory through numerical simulations performed on representative microstructures, consisting of random distributions of growing ferrite, bainite or martensite nuclei in an austenitic matrix. This allows to specifically investigate the effect of mechanical interactions between the nuclei (Barbe et al., 2007, 2008). To envisage large scale microstructures, with reasonable computation times and memory allocation, Moulinec and Suquet (1998)'s FFT-based numerical scheme is adopted. Indeed, such a scheme has repeatedly been shown to outperform the standard finite element method for elementary cell computations of periodic media, and has been successfully applied to a wide variety of problems and constitutive relations (see, among others, Lebensohn et al., 2008; Brenner et al., 2009; Lee et al., 2011; Lebensohn et al., 2012; Suquet et al., 2012; Otsuka et al., 2018).

The article is organized as follows. Section 2 expounds general hypotheses and notations. Section 3 presents the constitutive equations of the individual phases. Section 4 details Moulinec and Suquet (1998)'s numerical method, based on some FFT treatment of Lippmann-Schwinger's equation for periodic media, in the context of transformation plasticity - implying evolving microstructures. Section 5 presents a summary of El Majaty et al. (2018)'s new theory of transformation plasticity, based on the limit-analysis of a hollow sphere loaded both internally and externally. Section 6 is finally devoted to numerical results, and their comparison with the predictions of the limit-analysis-based theory.

\section{General hypotheses and notations}

In the whole paper, we use a geometrically linearized setting; the measures of strain and stress used are therefore the linearized strain and the Cauchy stress tensor.

We consider (Figure 1) a domain $\Omega$ made of a material containing two phases, one (denoted $\alpha$ ) ferritic, bainitic or martensitic, and the other (denoted $\gamma$ ) austenitic. A transformation (in practice induced by cooling) from the "mother" $\gamma$-phase to the "daughter" $\alpha$-phase may take place. When this occurs at a given point, the thermomechanical constants at this point vary from these values corresponding to the mother phase to those corresponding to the daughter phase.

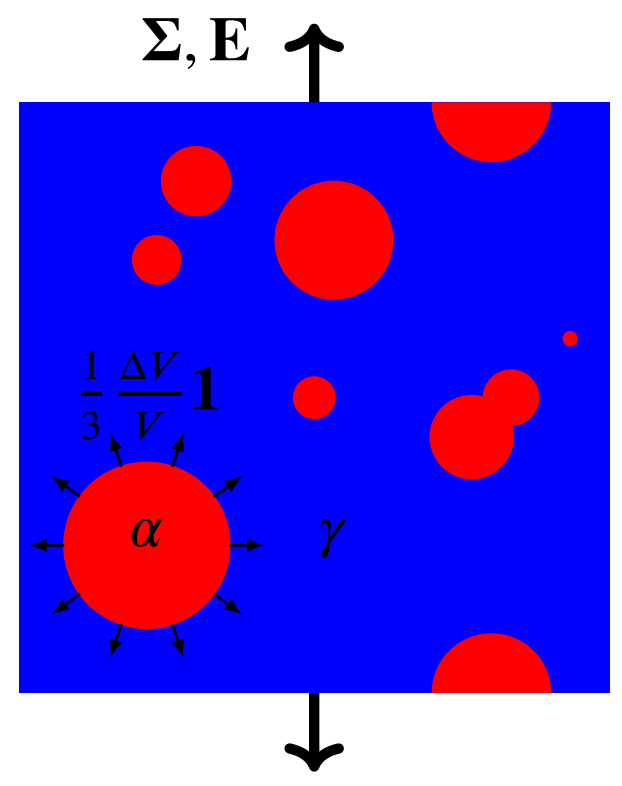

Figure 1: A 2D section of a periodic medium with cubic elementary cell, made of a $\gamma$-phase matrix containing growing nuclei of $\alpha$-phase, and subjected to some prescribed macroscopic stress $\boldsymbol{\Sigma}$ or $\mathbf{E}$.

At the local or microscopic scale, each point unambiguously belongs to one and one only of the two phases; the characteristic function of the daughter $\alpha$-phase is denoted $\zeta(\mathbf{x})$, so that $\zeta(\mathbf{x})=0$ if $\mathbf{x}$ lies in the $\gamma$-phase and $\zeta(\mathbf{x})=1$ if $\mathbf{x}$ lies in the $\alpha$-phase. The local or microscopic linearized strain and Cauchy stress tensors are denoted $\boldsymbol{\epsilon}(\mathbf{x})$ and $\sigma(\mathbf{x})$, respectively.

At the global or macroscopic scale, the domain $\Omega$ contains both phases; the volume fraction of the daughter $\alpha$ phase is denoted $f$ and is given by

$$
f=\langle\zeta(\mathbf{x})\rangle_{\Omega}
$$

where the symbol $\langle\cdot\rangle_{\Omega}$ denotes an average value over $\Omega$. The domain $\Omega$ is subjected to classical periodic boundary conditions. The global or macroscopic strain and stress tensors are denoted $\mathbf{E}$ and $\boldsymbol{\Sigma}$, respectively. These tensors satisfy the following relations ((Hill, 1967; Mandel, 1966)'s macrohomogeneity conditions):

$$
\boldsymbol{\Sigma}=\langle\boldsymbol{\sigma}(\mathbf{x})\rangle_{\Omega} ; \mathbf{E}=\langle\boldsymbol{\epsilon}(\mathbf{x})\rangle_{\Omega} ; \boldsymbol{\Sigma}: \mathbf{E}=\langle\boldsymbol{\sigma}(\mathbf{x}): \boldsymbol{\epsilon}(\mathbf{x})\rangle_{\Omega} .
$$

Time-derivatives of quantities will be denoted with an upper dot; thus, for instance, $\dot{\boldsymbol{\epsilon}}, \dot{\boldsymbol{\sigma}}, \dot{\mathbf{E}}, \dot{\boldsymbol{\Sigma}}$ will represent the rates of the microscopic strain and stress tensors and their macroscopic counterparts. This notation will classically be used to write the constitutive laws of the two phases; but since the behaviour of each of these phases will be assumed to be time-independent (no viscous effects), these laws could equally well be written in "incremental" form, thus replacing rates of all quantities by small increments. 


\section{Constitutive equations of the phases}

The behaviour of each phase is assumed to be thermoelasto-plastic. Thus the local strain $\boldsymbol{\epsilon}(\mathbf{x})$ can be split into thermal, elastic and plastic parts:

$$
\boldsymbol{\epsilon}(\mathbf{x})=\epsilon^{\mathrm{th}}(\mathbf{x})+\epsilon^{\mathrm{e}}(\mathbf{x})+\epsilon^{\mathrm{p}}(\mathbf{x}) .
$$

The "thermal" strain $\boldsymbol{\epsilon}^{\mathrm{th}}(\mathbf{x})$ is in fact related to both the thermal expansion and the volume change accompanying the transformation, according to the relation

$$
\epsilon^{\mathrm{th}}(\mathbf{x})=\left[\delta(\mathbf{x})\left(T(\mathbf{x})-T_{0}\right)+\beta(\mathbf{x})\right] \mathbf{1} .
$$

In this expression $\mathbf{1}$ is the unit second-rank tensor, $\delta(\mathbf{x})$ the local thermal expansion coefficient, $T_{0}$ and $T(\mathbf{x})$ the "reference" and actual temperatures, and $\beta(\mathbf{x})$ a scalar related to the difference of specific volume $\frac{\Delta V}{V}$ between the phases at the temperature $T_{0}$, defined as:

$$
\beta(\mathbf{x})=\frac{1}{3} \frac{\Delta V}{V} \zeta(\mathbf{x}) .
$$

(Thus $\beta(\mathbf{x})$ is zero in the $\gamma$-phase and equal to $\frac{1}{3} \frac{\Delta V}{V}$ in the $\alpha$-phase). Note that according to equations (4) and (5), the thermal strain is zero in the $\gamma$-phase at the temperature $T_{0}$, implying that such conditions define the "reference state" for the measure of strains.

The elastic strain $\epsilon^{\mathrm{e}}(\mathbf{x})$ is related to the stress $\sigma(\mathbf{x})$ through the elasticity law:

$$
\sigma(\mathbf{x})=\mathbf{C}(\mathbf{x}): \epsilon^{\mathrm{e}}(\mathbf{x})
$$

where $\mathbf{C}(\mathbf{x})$ denotes the local fourth-rank elastic stiffness tensor. In this paper, we disregard both the variation of $\mathbf{C}(\mathbf{x})$ within each phase (arising from the anisotropy of grains and their varying orientations), and the difference of elastic moduli between the phases; thus $\mathbf{C}(\mathbf{x})$ is identified to $\mathbf{C}^{0}$, the global, isotropic elastic stiffness tensor.

Finally the plastic strain rate is given by the classical equations of time-independent, ideal-plasticity, for an (isotropic) von Mises criterion and a plastic flow rule following Hill's normality property. Thus the yield criterion reads

$$
\phi(\sigma(\mathbf{x}))=\sqrt{3 J_{2}(\mathbf{x})}-\sigma_{Y}(\mathbf{x}) \leq 0
$$

where $J_{2}(\mathbf{x})$ is the second (deviatoric) stress invariant, and $\sigma_{Y}(\mathbf{x})$ the local yield stress (depending on the phase the point $\mathbf{x}$ belongs to). Also, the flow rule reads

$$
\dot{\boldsymbol{\epsilon}}^{\mathrm{p}}(\mathbf{x})=\dot{\lambda} \frac{\partial \phi}{\partial \sigma}(\sigma(\mathbf{x}))=\dot{\lambda}(\mathbf{x}) \frac{3}{2 \sigma_{Y}(\mathbf{x})} \mathbf{s}(\mathbf{x})
$$

where $\mathbf{s}(\mathbf{x})$ denotes the local stress deviator, and $\dot{\lambda}(\mathbf{x})$ the local plastic multiplier, satisfying the Kühn-Tucker conditions:

$$
\phi(\sigma(\mathbf{x})) \leq 0 \quad ; \quad \dot{\lambda}(\mathbf{x}) \geq 0 \quad ; \quad \phi(\sigma(\mathbf{x})) \dot{\lambda}(\mathbf{x})=0 .
$$

Since the plastic flow rule inevitably involves the rate (or increment) of the plastic strain, it is necessary to express the constitutive equations in rate (or incremental) form. Equation (3) thus becomes

$$
\dot{\boldsymbol{\epsilon}}(\mathbf{x})=\dot{\boldsymbol{\epsilon}}^{\mathrm{th}}(\mathbf{x})+\dot{\boldsymbol{\epsilon}}^{\mathrm{e}}(\mathbf{x})+\dot{\boldsymbol{\epsilon}}^{\mathrm{p}}(\mathbf{x}) .
$$

In this expression:

- $\dot{\boldsymbol{\epsilon}}^{\mathrm{p}}(\mathbf{x})$ is given by equation (8).

- $\dot{\boldsymbol{\epsilon}}^{\mathrm{th}}(\mathbf{x})$ is given by

$$
\dot{\boldsymbol{\epsilon}}^{\mathrm{th}}(\mathbf{x}) \simeq \dot{\beta}(\mathbf{x}) \mathbf{1} \quad, \quad \dot{\beta}(\mathbf{x}) \simeq \frac{1}{3} \frac{\Delta V}{V} \dot{\zeta}(\mathbf{x})
$$

where the variation of volume due to the variation of temperature has been neglected compared to that due to the transformation $(\delta(\mathbf{x}) \dot{T}(\mathbf{x}) \ll \dot{\beta}(\mathbf{x}))$, and the dependence of the difference of specific volume between the phases upon temperature has also been disregarded $\left(\frac{d}{d t} \frac{\Delta V}{V} \simeq 0\right)$. Note also that the derivative $\dot{\zeta}(\mathbf{x})$ in the second of equations (11) must be understood in the sense of generalized functions since the function $\zeta(\mathbf{x})$ only takes the values 0 and 1 .

- $\dot{\boldsymbol{\epsilon}}^{\mathrm{e}}(\mathbf{x})$ is given by

$$
\dot{\boldsymbol{\sigma}}(\mathbf{x})=\mathbf{C}^{0}: \dot{\boldsymbol{\epsilon}}^{\mathrm{e}}(\mathbf{x})
$$

where the dependence of the overall elastic stiffness tensor $\mathbf{C}^{0}$ upon temperature has been neglected $\left(\dot{\mathbf{C}}^{0} \simeq\right.$ $\mathbf{0})$.

(Note that the approximations made to get equations (11) and (12) are equivalent to artificially considering the transformation as isothermal).

\section{Remarks on the hypotheses made.}

1. In reality, and especially in the case of martensitic transformations, the transformation strain does not include only an isotropic part $\beta(\mathbf{x}) \mathbf{1}$ but also a deviatoric part. In principle, accounting for such a deviatoric part would not raise any problem of principle, either in the theoretical description or the numerical simulations. The difficulty, however, would be to properly account for the partial or total compensation of the deviatoric transformation strain from one point to a neighbouring one (that is, for the self-accommodation of the transforming regions). In this work we follow Greenwood and Johnson (1965)'s point of view, which consisted in accounting for the sole part of the transformation strain that cannot average out to zero, that is its isotropic part $\beta(\mathbf{x}) \mathbf{1}$.

2. Replacing the local elastic stiffness tensor $\mathbf{C}(\mathbf{x})$ with the overall tensor $\mathbf{C}^{0}$ seems reasonable insofar as elasticity is not expected to play a major role in transformation plasticity.

3. With regard to plasticity, the use of the global isotropic model of von Mises instead of some local crystal plasticity model is more debatable. We again follow here Greenwood and Johnson (1965)'s approach, in which crystal plasticity effects were considered as secondorder. Such effects (in the context of transformation plasticity) were considered in the work of Otsuka et al. (2018), to which the reader is referred on this topic. 
4. Making the assumption of ideal-plasticity means neglecting all effects tied to strain hardening; this is acceptable in a first approximation, consideration of such effects being postponed to some later work. This simplification is made to eliminate several complicating factors, and notably the possible memory/recovery of hardening during the transformation (tied to the modification of the arrangement of dislocations arising from the structural change).

5. A similar remark applies to the neglect of viscous effects (which are bound to be of some importance at the temperatures of interest).

\section{The FFT-based numerical scheme in the context of transformation plasticity}

Moulinec and Suquet (1998)'s FFT-based numerical method iteratively solves the integral Lippmann-Schwinger (LS) equation, the solution of which represents the strain field in a fictitious homogeneous medium with a heterogeneous "polarization stress field". In the present study, periodic elastoplastic problems with prescribed growth kinetics of nuclei are considered (Figure 2).

\subsection{Lippmann-Schwinger's equation for periodic media}

Combination of equations (10) and (12) yields

$$
\dot{\sigma}(\mathbf{x})=\mathbf{C}^{0}: \dot{\epsilon}(\mathbf{x})+\dot{\boldsymbol{\tau}}(\mathbf{x})
$$

where $\boldsymbol{\tau}(\mathbf{x})$ is a "polarization stress rate field" given by

$$
\dot{\boldsymbol{\tau}}(\mathbf{x})=-\mathbf{C}^{0}:\left[\dot{\boldsymbol{\epsilon}}^{\mathrm{th}}(\mathbf{x})+\dot{\boldsymbol{\epsilon}}^{\mathrm{p}}(\mathbf{x})\right] .
$$

In the solution of the quasi-static global problem thus defined, the strain rate may be expressed as a function of the polarization field, through the LS integral equation involving the Green operator $\boldsymbol{\Gamma}^{0}$ associated to the stiffness tensor $\mathbf{C}^{0}$ :

$$
\dot{\boldsymbol{\epsilon}}(\mathbf{x})=-\left(\boldsymbol{\Gamma}^{0} * \dot{\boldsymbol{\tau}}\right)(\mathbf{x})+\dot{\mathbf{E}}
$$

where $\dot{\mathbf{E}}$ denotes the average of the strain rate $\dot{\boldsymbol{\epsilon}}(\mathbf{x})$ over any elementary cell of the periodic medium considered. The Fourier transform of the LS equation reads

$$
\hat{\hat{\epsilon}}(\xi)=-\hat{\boldsymbol{\Gamma}}^{0}(\xi): \hat{\boldsymbol{\tau}}(\xi) \quad \forall \xi \neq \mathbf{0} \quad, \quad \hat{\hat{\epsilon}}(\mathbf{0})=\dot{\mathbf{E}}
$$

where $\hat{\boldsymbol{\Gamma}}^{0}$ denotes the Green operator in Fourier's space (this operator is explicitly known for an isotropic $\mathbf{C}^{0}$ ), and $\boldsymbol{\xi}$ the wavevector. Note that formula (16) does not directly provide the value of $\hat{\dot{\epsilon}}(\xi)$ but rather an equation on this quantity, since the polarization rate field $\hat{\boldsymbol{t}}(\boldsymbol{\xi})$ in the right-hand side depends upon $\hat{\hat{\epsilon}}(\xi)$, through equation (14) plus the thermoelasto-plastic constitutive law which relates $\dot{\boldsymbol{\epsilon}}^{\mathrm{p}}(\mathbf{x})$ to $\dot{\boldsymbol{\epsilon}}(\mathbf{x})$. For each time increment, the problem is solved iteratively by alternating between the spatial domain for the solution of the constitutive equations (calculation of $\dot{\boldsymbol{\epsilon}}^{\mathrm{p}}(\mathbf{x})$ from $\dot{\boldsymbol{\epsilon}}(\mathbf{x})$ ), and the Fourier domain for the application of equation (16). Note that this permits to solve only local equations in each domain. The case of an imposed macroscopic stress may be handled with an additional step, aimed at computing the corresponding macroscopic strain rate tensor (Moulinec and Suquet, 1998).

The facilities at the HPCaVe Center at Sorbonne Université are used for these calculations.

\subsection{Microstructure evolution \\ 4.2.1. Preliminary considerations}

Two important preliminary remarks are in order. First, in all numerical simulations discussed below, the sequence of successive configurations of the two phases, defining the geometric evolution of the transformation, will result from some pre-specified rules independent of the internal stresses and plastic strains generated by the external load and the transformation. In other words, the transformation will be considered to be governed by given external factors, but not by the a priori unknown mechanical fields.

Second, the numerical model depicted below will involve no absolute characteristic lengthscale (all distances could be multiplied by a given, fixed factor without changing the results). This means disregarding, among other things, the initial "critical" nucleus size introduced by classical nucleation theory from thermodynamic considerations.

Both of these approximations will be justified a posteriori by the observation that transformation plasticity is essentially independent of the details of the geometric modelling of the transformation.

To model the $\gamma \rightarrow \alpha$ transformation in successive timesteps, we "switch", between some discretized instant $t$ and the next one $t+\Delta t$, a certain number of pre-specified voxels from the $\gamma$ - to the $\alpha$-phase; in practice this means changing the values of $\beta(\mathbf{x})$ and $\sigma_{Y}(\mathbf{x})$ at these points from these of the $\gamma$-phase to those of the $\alpha$-phase. The voxels thus "transformed" at each step are selected in such a way as to simulate the nucleation and growth of a set of spherical nuclei. Figure 2 illustrates such a process by showing the geometrical distribution of the two phases at two different stages of the transformation, in a typical simulation.

\subsubsection{The local nucleation and growth processes}

During any time-step $[t, t+\Delta t]$, the transformation may proceed in two ways:

- Through growth of pre-existing $\alpha$-phase nuclei, via superposition of an external concentric layer with a certain thickness. The value of this thickness is set here to the size of a voxel.

- Through nucleation of new nuclei of $\alpha$-phase, chosen at random within the entire un-transformed zone of the elementary cell considered. Again, the initial size of new nuclei is set to the size of a voxel.

Of course, various combinations of these two mechanisms are possible.

Remarks. 


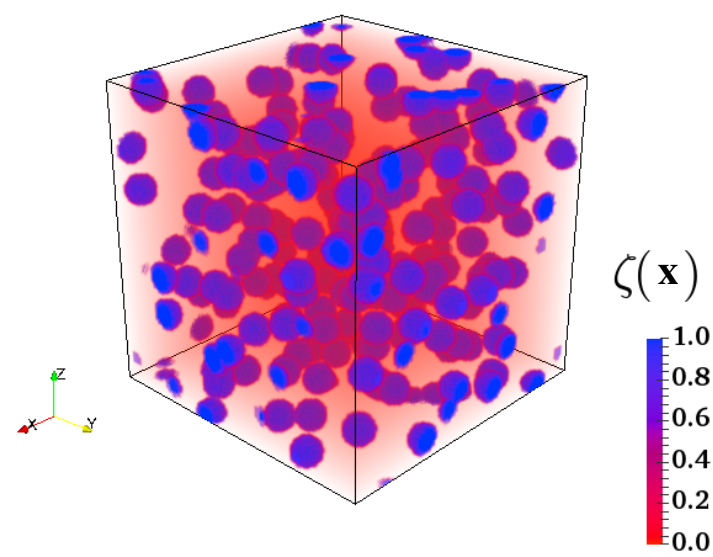

(a) $\simeq 10 \%$ of $\alpha$-phase

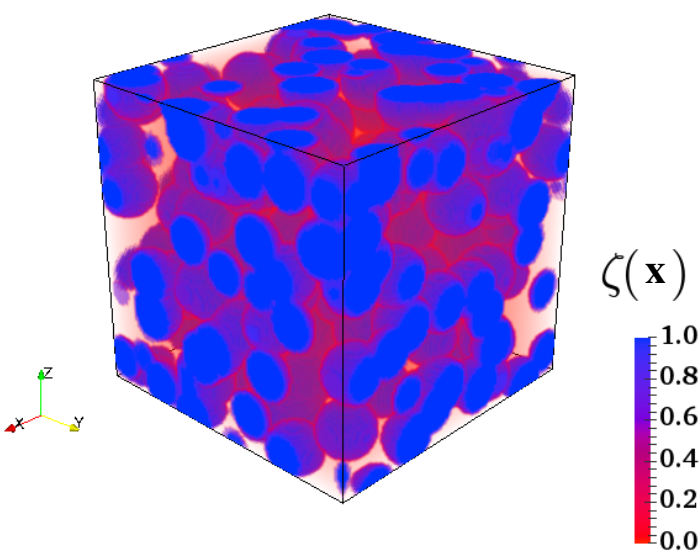

(b) $\simeq 50 \%$ of $\alpha$-phase

Figure 2: Spherical growth of $\alpha$-phase nuclei. (For the explanation of values of $\zeta(\mathbf{x})$ differing from 0 and 1 , see subsubsection 4.2.4).

1. Voxels potentially available for transformation, be it through growth of pre-existing nuclei or nucleation of new ones, are rejected for transformation if they happen to fall within the already transformed region.

2. Growing nuclei of $\alpha$-phase are allowed to overlap (this means accounting, albeit in a rough way, for coalescence of nuclei). This occurs more and more in time, as a natural result of the growth process; see Figure 2 which compares the geometric configurations of the phases at two distinct stages of the transformation, in a typical simulation.

\subsubsection{Kinetic model of nucleation and growth}

The following features of the modelling of nucleation and growth processes are noteworthy:

- Nucleation occurs randomly and homogeneously within the entire un-transformed part of the material (homogeneous nucleation).

- The rate of growth of nuclei is the same in all directions (spherical growth).

- The radial rate of growth of nuclei is constant during the entire transformation.

With these three assumptions, the overall transformed volume fraction $f=\langle\zeta(\mathbf{x})\rangle_{\Omega}$ should follow the Johnson-MehlAvrami-Kolmogorov (JMAK) equation for solid-state transformations at constant temperature:

$$
f(t)=1-\exp \left(-K t^{n}\right)
$$

where the symbol $t$ denotes time, the parameter $K$ governs the overall growth rate, and the exponent $n$ is connected to the type of nucleation:
- For instantaneous nucleation (IN), all nuclei are "activated" (transformed from the $\gamma$-phase to the $\alpha$-phase) right from the beginning of the transformation; in other words, the nucleation and growth processes are disjoint in time: nucleation occurs first without any growth, then growth takes over without any further nucleation. The value of the JMAK exponent $n$ is then 3 .

- For sporadic nucleation (SN), new $\alpha$-phase nuclei are "activated" throughout the transformation. This means nucleation and growth occur simultaneously throughout the entire transformation. The value of $n$ is then 4.

It is worth noting here that the question of whether the transformations simulated here (following the geometric rules expounded above) do or do not obey a JMAK-type kinetic equation, is in some sense irrelevant in the present context. Indeed, since the constitutive laws adopted for the individual phases are independent of time, what really matters is only the succession of geometric configurations of the two phases, not the instants at which these configurations occur. However the verification that the transformations simulated as explained above do obey a JMAK-type kinetic equation is useful for two purposes: (i) validate the physical soundness of the numerical procedure proposed; (ii) show that such a procedure would remain reasonable for future studies incorporating time-dependent constitutive laws of the phases.

Before discussing this topic in detail, it is necessary to introduce some conventions and notations:

- The side of the cubical elementary cell considered is taken to be of unit length. Thus the volume of the cell is unity.

- The length $\Delta t$ of each time-step (corresponding to one 
transformation step) is also considered to be unity. The "time" $t$ is thus identical to the number of successive transformation steps.

- The total number of voxels in the cell is denoted $v^{3}$ (there are thus $v$ voxels in each direction).

- The number of " $\alpha$-phase voxels" appearing instantaneously at the beginning of the transformation is denoted $N_{0}$. The "density of instantaneous nucleation" is $Z_{0} \equiv N_{0} / v^{3}$; it represents the volume fraction of voxels transformed instantaneously.

- The number of new $\alpha$-phase voxels generated by nucleation at each transformation step is denoted $\dot{N}_{0}{ }^{2}$ The "density of sporadic nucleation" is $\dot{Z}_{0} \equiv \dot{N}_{0} / v^{3}$; it represents the volume fraction of newly nucleated voxels at each step.

- The "radial growth rate" of the nuclei is denoted $G$; since at each transformation step, of duration $\Delta t=1$, one voxel, of size $1 / v$, is added to each nucleus in the radial direction, the value of this growth rate is $G=$ $1 / v$.

Numerical simulations were performed, with the values $N_{0}=10,10^{2}, 10^{3}$ and $\dot{N}_{0}=10,10^{2}, 10^{3}$, in a cell discretized with $v^{3}=100^{3}$ voxels; therefore the values of the instantaneous nucleation density were $Z_{0}=10^{-5}, 10^{-4}, 10^{-3}$, and those of the sporadic nucleation density, $\dot{Z}_{0}=10^{-5}, 10^{-4}$, $10^{-3}$, respectively.

Numerical plots of $\ln (-\ln (1-f))$ versus $\ln (t)$, shown in Figure 3a and Figure 4a, were used to determine values of the JMAK parameters $n$ and $K$ matching best the simulated transformations. Table 1 shows the results obtained and compares them to the theoretical values. As can be seen, the simulations lead to numerical JMAK parameters $n$ and $K$ consistent with the theory for both types of nucleation, instantaneous and sporadic. (The theoretical $K$ value is equal to $4.19 \times 10^{-5}, 4.19 \times 10^{-4}, 4.19 \times 10^{-3}$ and $1.0472 \times 10^{-5}$, $1.0472 \times 10^{-4}, 1.0472 \times 10^{-3}$ for instantaneous and sporadic nucleation respectively, with $Z_{0}$ or $\dot{Z}_{0}$ equal to $10^{-5}$, $10^{-4}, 10^{-3}$ respectively). The slight discrepancies in Table 1 may be due to the representation of the microstructure in the simulations, which does not exactly match the theoretical hypotheses (since it is periodic and discrete, instead of infinite and continuous).

Figure $3 \mathrm{~b}$ and Figure $4 \mathrm{~b}$ illustrate the excellent agreement obtained for the transformation kinetics. These figures display three types of curves: (i) "Numerical", showing the simulated kinetics; (ii) "Theoretical", showing the kinetics corresponding to the JMAK model, with the theoretical parameters shown in Table 1; (iii) "Semi-analytical", showing the kinetics corresponding to the JMAK model, but now with the parameters $n$ and $K$ determined from the simulations, again shown in Table 1.

\footnotetext{
${ }^{2}$ In this definition one does not account for the fact that some of these voxels must in fact be eliminated, because they happen to fall within the already transformed region; see subsubsection 4.2.2.
}

Table 1

Values of JMAK parameters $n$ and $K$.

\begin{tabular}{lcc|ccc}
\hline \hline & \multicolumn{2}{c}{ IN } & \multicolumn{2}{c}{ SN } \\
\hline & $n$ & $K$ & $n$ & $K$ \\
& \multicolumn{5}{l}{ KN } \\
\hline Theoretical & 3 & $4 \pi N_{0} G^{3} / 3$ & 4 & $\pi \dot{N}_{0} G^{3} / 3$ \\
Num. $N_{0}, \dot{N}_{0}=10$ & 3.18 & $2.67 \times 10^{-5}$ & 3.78 & $2.18 \times 10^{-5}$ \\
Num. $N_{0}, \dot{N}_{0}=100$ & 3.13 & $3.15 \times 10^{-4}$ & 3.58 & $2.72 \times 10^{-4}$ \\
Num. $N_{0}, \dot{N}_{0}=1000$ & 3.00 & $3.40 \times 10^{-3}$ & 3.65 & $2.60 \times 10^{-3}$ \\
\hline
\end{tabular}

\subsubsection{Time sub-stepping}

In fact, in what precedes the description of the numerical procedure has been slightly simplified, for clarity of the presentation. But in reality, transforming each voxel from the $\gamma$-phase to the $\alpha$-phase in just one time-step, as suggested above, reveals impossible, because the large and brutal variation of the thermal strain in the region transformed betwen times $t$ and $t+\Delta t$ makes convergence of the global iterations (aimed at solving the global nonlinear mechanical problem between these instants) impossible.

For this reason each time-step $[t, t+\Delta t]$, corresponding to a given transformation step, that is to transformation of a given region, is divided into a number of substeps; in practice 5. During the time-interval $[t, t+\Delta t]$, the region to be transformed is in fact transformed gradually, using the substeps to do so: in this region, the function $\zeta(\mathbf{x})$ is artificially ascribed the successive values $0,0.2,0.4,0.6,0.8,1$ instead of just 0 and 1 ; and the parameter $\beta(\mathbf{x})$ and the yield stress $\sigma_{Y}(\mathbf{x})$ are also varied gradually, according to the value of $\zeta(\mathbf{x})$. (Of course, in the complementary region not undergoing any transformation during the time-interval $[t, t+\Delta t]$, $\beta(\mathbf{x})$ and $\sigma_{Y}(\mathbf{x})$ remain constant at each point throughout this time-interval).

The parameter $\beta(\mathbf{x})$ may be taken in a natural way as a linear function of $\zeta(\mathbf{x})$. However, how the yield stress $\sigma_{Y}(\mathbf{x})$ should be considered to vary with $\zeta(\mathbf{x})$ is a less trivial matter. Indeed numerical experience shows that the transformation plastic strain rate obtained after a complete transformation depends, to some extent, on the way $\sigma_{Y}(\mathbf{x})$ is considered to vary with $\zeta(\mathbf{x})$; see Appendix B.1 for some details on this topic. In all simulations presented below (except a few ones discussed in Appendix B.1), the yield stress has been assumed to retain the value corresponding to the mother, $\gamma$ phase until $\zeta(\mathbf{x})$ reaches the value 0.8 ; and then to evolve linearly toward the value corresponding to the daughter, $\alpha$ phase over the interval $\zeta(\mathbf{x}) \in[0.8,1]$. This evolution of the yield stress, which implies a low resistance to stresses during the major part of the transformation, seems reasonable since the motion of atoms during the structural change must make the material prone to yielding.

Figure 5 illustrates the evolutions thus assumed for both the parameter $\beta(\mathbf{x})$ and the yield stress $\sigma_{Y}(\mathbf{x})$ at a given voxel, as functions of the substeps of the time-step during which this voxel is transformed. 


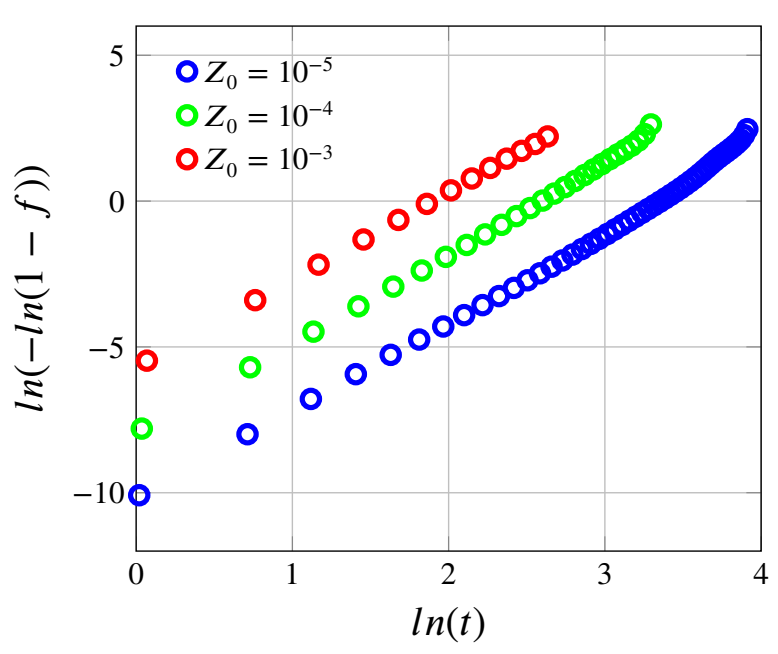

(a) Fit of numerical coefficients of the JMAK model

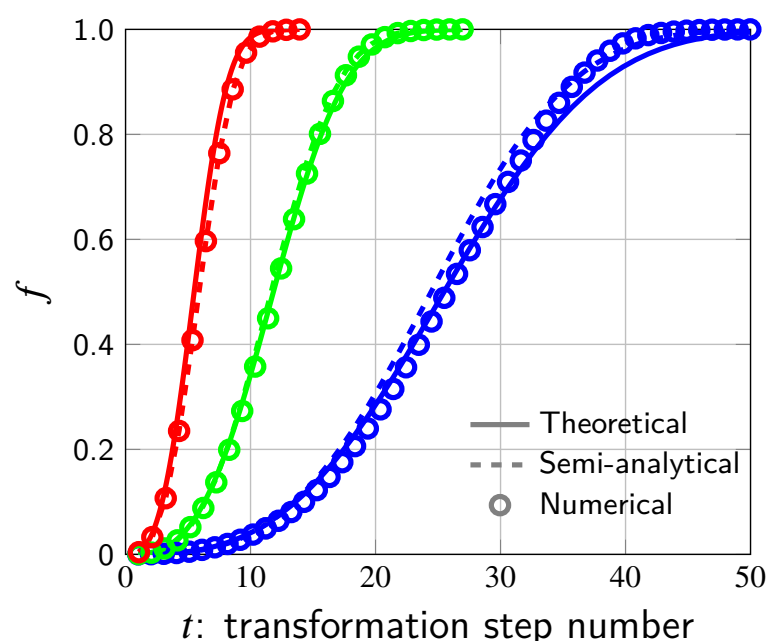

(b) Extent of transformation

Figure 3: Comparison between the predictions of the JMAK model and the results of instantaneous nucleation simulations, for three values of the instantaneous nucleation density $Z_{0}$ (IN).

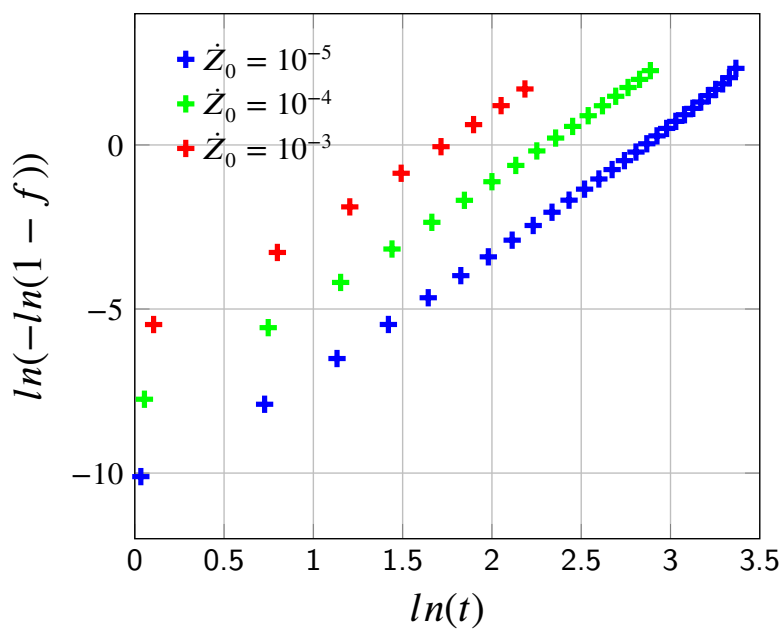

(a) Fit of numerical coefficients of the JMAK model

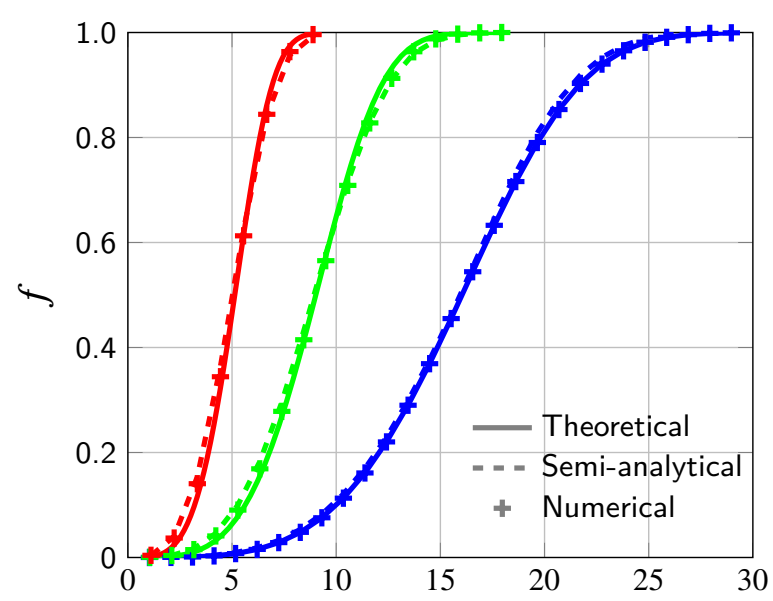

$t$ : transformation step number

(b) Extent of transformation

Figure 4: Comparison between the predictions of the JMAK model and the results of sporadic nucleation simulations, for three values of the sporadic nucleation density $\dot{Z}_{0}(\mathrm{SN})$.

Table 2

Material properties (A533 steel).

\begin{tabular}{cccccc}
\hline \hline & $E(\mathrm{GPa})$ & $v$ & $\sigma_{Y}(\mathrm{MPa})$ & $\beta$ & $(\zeta)$ \\
\hline$\gamma$-phase & 182 & 0.3 & 145 & 0 & $(0)$ \\
$\alpha$-phase & 182 & 0.3 & 950 & 0.0084 & $(1)$ \\
\hline
\end{tabular}

\subsubsection{Thermomechanical properties of the phases}

The transformation studied in all simulations discussed below is the martensitic transformation of the A533 steel. All thermal and mechanical properties are displayed in Table 2 .

\subsection{Preliminary study of dispersion effects}

Values of the transformation plastic strain after complete transformation obtained numerically are dispersed for various reasons. A preliminary study of dispersion effets, according to their origin, is therefore in order.

\subsubsection{Effect of the realization of the microstructure}

Different possible "realizations" of the random distribution of positions of nuclei lead to different transformation plastic strains after complete transformation. We therefore perform a study aimed at determining the number of realizations required to obtain an accurate (averaged) estimate of the transformation plastic strain. This is done in the special case of a zero applied macroscopic stress, in which the 
FFT-based simulations of transformation plasticity
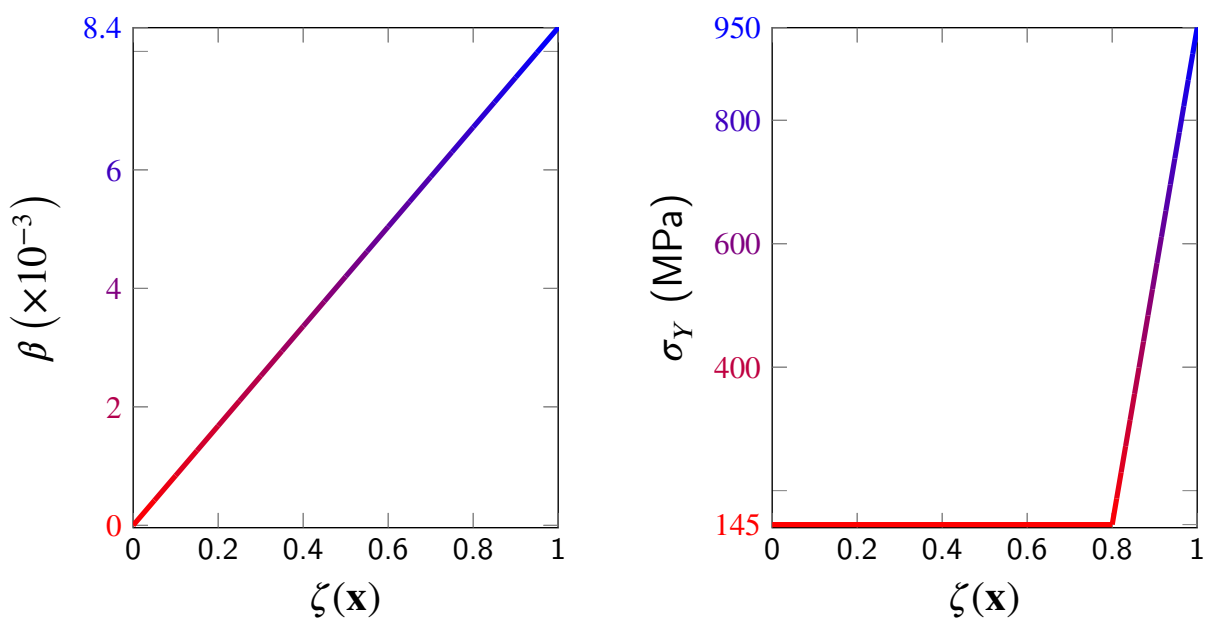

Figure 5: Evolutions of the thermal strain and yield stress during the transformation process.

\section{Table 3}

Statistics of the histograms of the overall strain shown in Figure 6: Mean (M) - Standard deviation (SD) - Coefficient of variation $=\mathrm{SD} / \mathrm{M}(\mathrm{CV})$.

\begin{tabular}{lccr}
\hline \hline & $Z_{0}=10^{-5}$ & $Z_{0}=10^{-4}$ & $Z_{0}=10^{-3}$ \\
\hline $\mathrm{M}$ & $8.4 \times 10^{-3}$ & $8.4 \times 10^{-3}$ & $8.4 \times 10^{-3}$ \\
$\mathrm{SD}$ & $7.7 \times 10^{-4}$ & $2.5 \times 10^{-4}$ & $0.9 \times 10^{-4}$ \\
$\mathrm{CV}$ & $9.2 \%$ & $3.0 \%$ & $1.1 \%$ \\
\hline
\end{tabular}

theoretical transformation plastic strain is known to be zero for spherical (isotropic) growth of nuclei; the total strain after complete transformation then reduces to that part arising from the volume change induced by the transformation, $\frac{1}{3} \frac{\Delta V}{V} \mathbf{1}$ (Leblond et al., 1989).

Envisageable situations are too numerous to be all considered; we therefore decide to focus on the sole case of instantaneous nucleation (IN). FFT simulations are thus performed for instantaneous nucleation densities $Z_{0}=10^{-5}$, $10^{-4}, 10^{-3}$. Seven realizations are considered for each density. Figure 6 displays the histograms of the overall strain components in three orthogonal directions. (The results of individual realizations are plotted in grey). As expected, the fluctuations of the overall strain arising from the fluctuations of the spatial distribution of nuclei decrease when the number of nuclei increases; see Figure 6 and Table 3. Note also that when the density $Z_{0}$ increases, the number of transformation steps decreases, which probably makes the simulations less representative.

These results lead to the decision of performing, in each case considered, averages over 5 realizations of cells discretized with $v^{3}=100^{3}$ voxels and containing $N_{0}=100$ nuclei. For each realization, the overall isotropy of the microstructure is assessed by checking the quasi-spherical character of the autocorrelation function: see Figure $7 .^{3}$

\footnotetext{
${ }^{3}$ The autocorrelation technique calculates the similarity of an image with itself when gradually displaced in all directions. If the pattern of the
}

As a complement, Figure 8 shows the distribution of the equivalent strain rate in the austenitic phase at two stages of the transformation. This figure emphasizes the effect of interactions between $\alpha$-phase nuclei upon strain heterogeneities in the surrounding $\gamma$-phase.

\subsubsection{Effect of the nature (instantaneous/sporadic) of nucleation}

A preliminary remark is that although a number of theoretical works were based on the hypothesis that no new nucleus appears once the growth of the daughter-phase has started (IN), see for instance (Kempen et al., 2002), some experimental works have shown that new nuclei continue to appear during the entire transformation ( $\mathrm{SN})$ : see notably the microtomographic studies of Offerman et al. (2003) and (Offerman et al., 2006). It is therefore necessary to envisage SN, in addition to IN.

Figure 9 displays the evolutions of the transformation plastic strain versus the progress of the transformation, for both IN and SN, for various values of the instantaneous nucleation density $\left(Z_{0}=10^{-5}, 10^{-4}, 10^{-3}\right)$ and the sporadic nucleation density $\left(\dot{Z}_{0}=10^{-5}, 10^{-4}, 10^{-3}\right)$. In all cases an overall tensile stress of $80 \mathrm{MPa}$ was applied on the cell in the direction $x_{3}$. One observes on this figure that neither the value of the instantaneous or sporadic nucleation density, nor the nature (instantaneous versus sporadic) nature of nucleation have an important influence upon the transformation plastic strain.

\subsubsection{Other sources of dispersion}

Other dispersion effects are discussed in appendices: appendix $\mathrm{A}$ is devoted to the effect of the possible heterogeneous character of nucleation, and appendix $\mathrm{B}$ to that of certain parameters of the numerical scheme used to represent the transformation.

autocorrelation function is spherical, the structure is globally isotropic. 
(a) Histogram - $Z_{0}=10^{-5}$

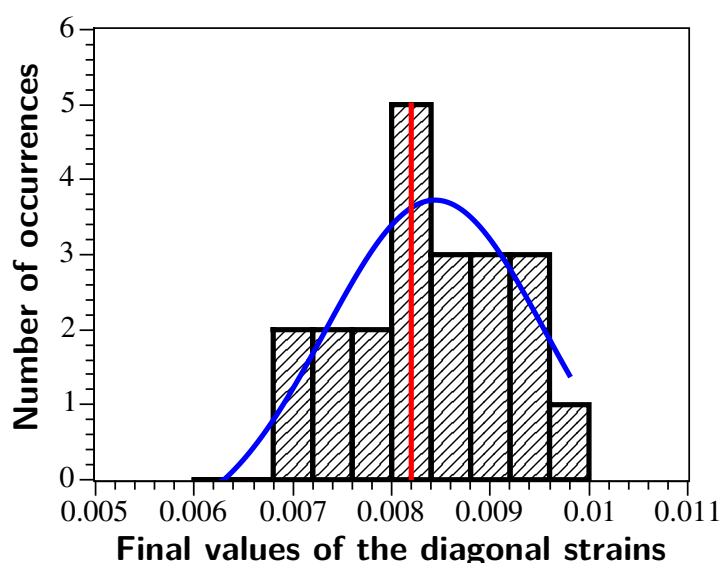

(c) Histogram - $Z_{0}=10^{-4}$

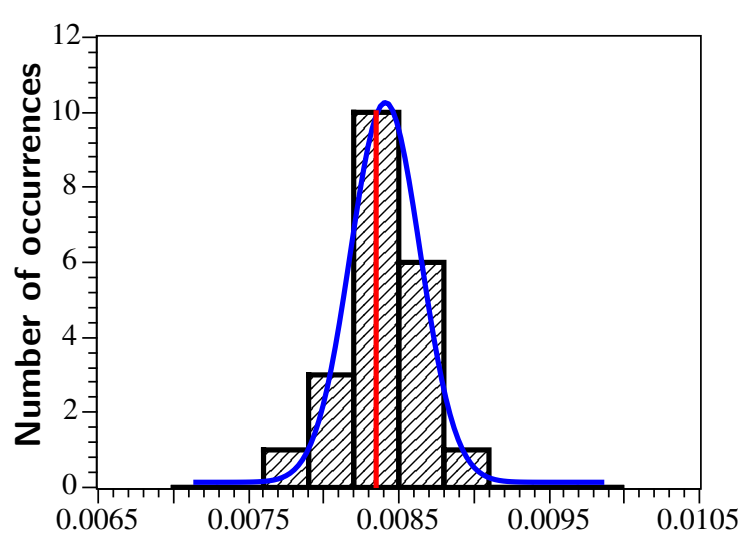

Final values of the diagonal strains

(e) Histogram $-Z_{0}=10^{-3}$

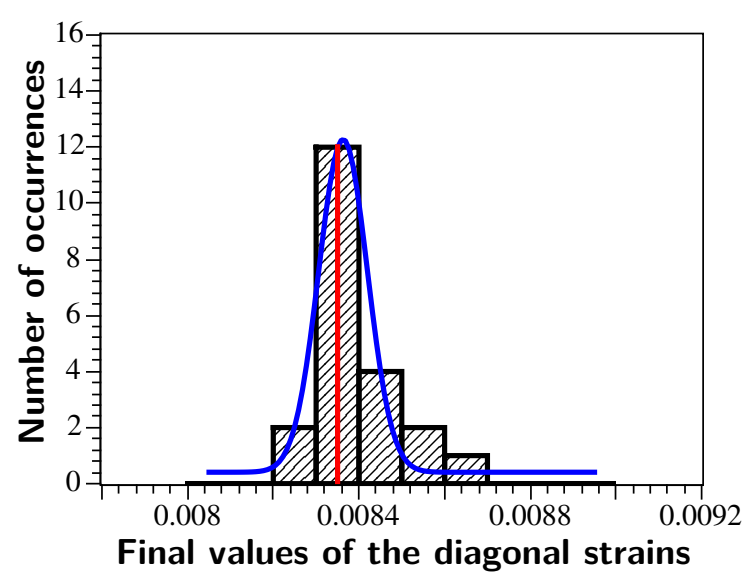

(b) Mean of the diagonal strains $-Z_{0}=10^{-5}$

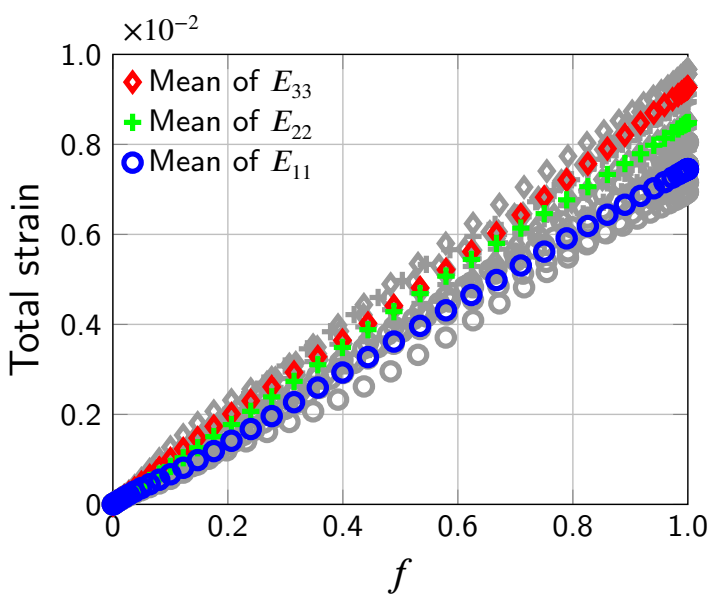

(d) Mean of the diagonal strains $-Z_{0}=10^{-4}$

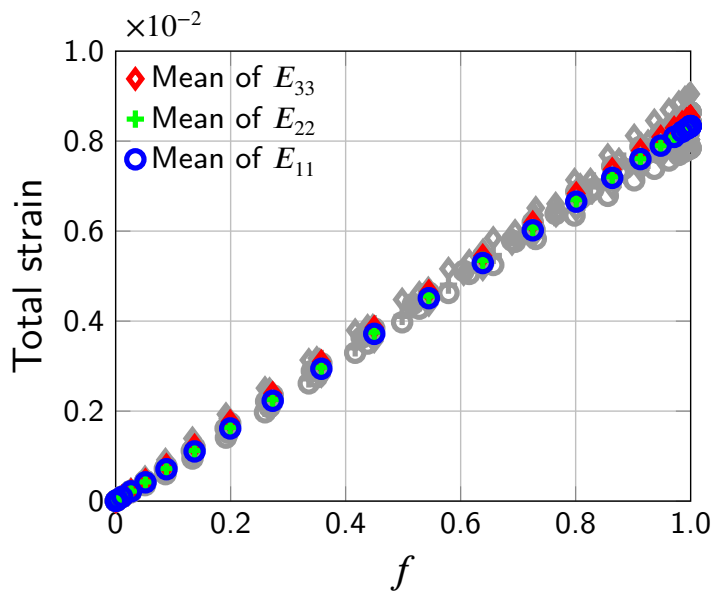

(f) Mean of the diagonal strains $-Z_{0}=10^{-3}$

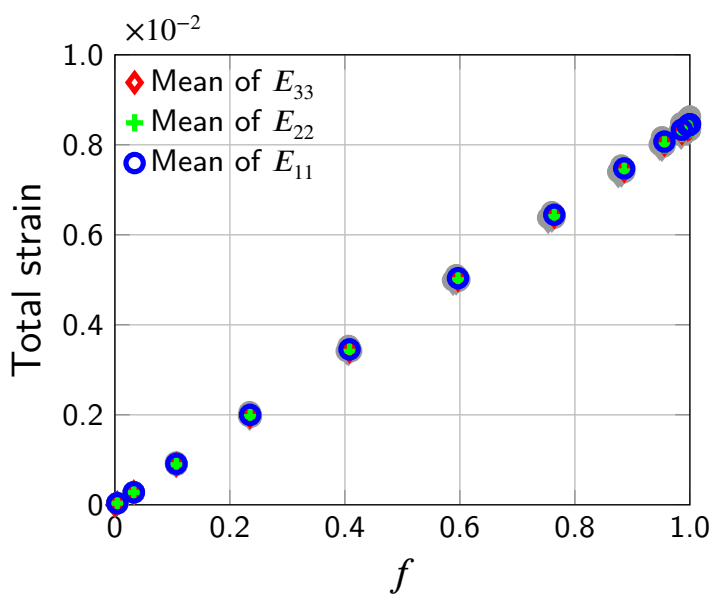

Figure 6: Histograms of the diagonal components of the overall strain after complete transformation, and evolution of these components during the transformation, for various instantaneous nucleation densities $Z_{0}$. No macroscopic stress applied.

\section{A summary of a limit-analysis-based theory of transformation plasticity}

An original approach (El Majaty et al., 2018) was proposed very recently in order to overcome some shortcomings 

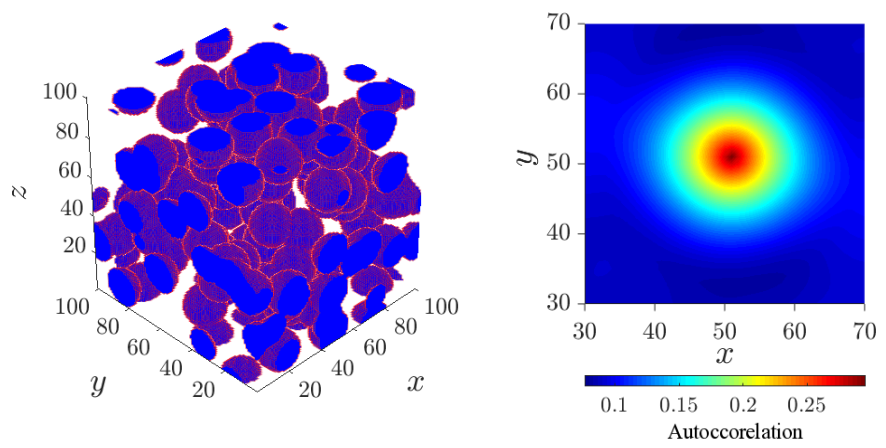
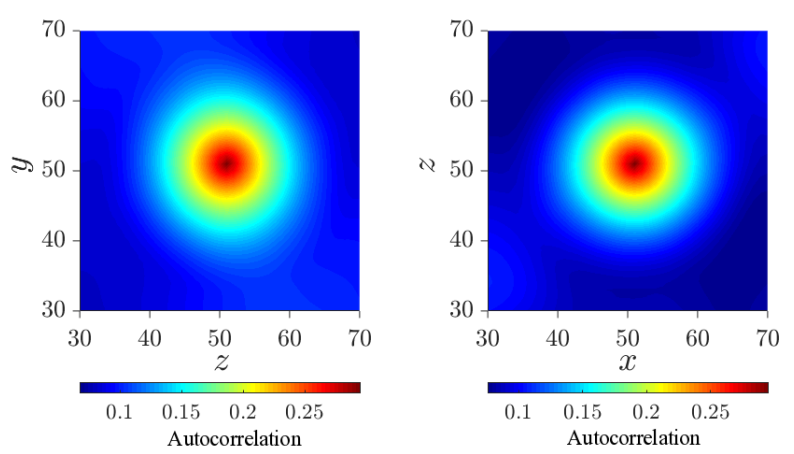

Figure 7: Autocorrelation function of an evolving microstructure at $f=30 \%$. Microstructure image and isovalues of the function in three orthogonal planes.

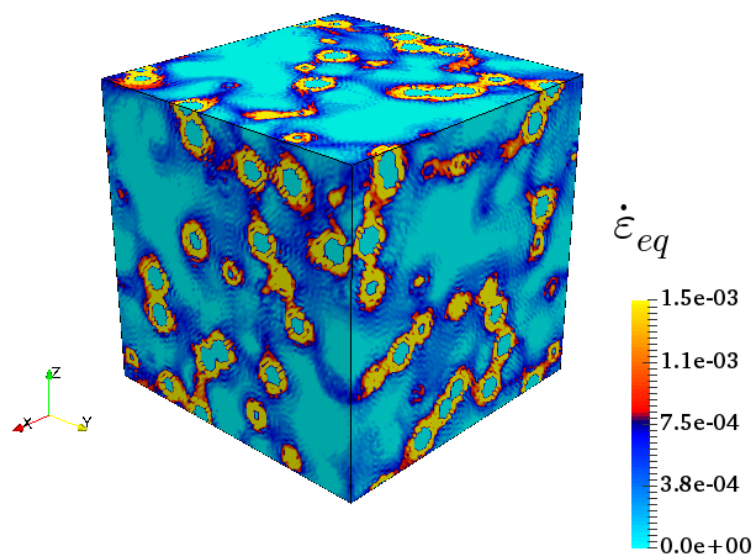

(a) $\simeq 10 \%$ of $\alpha$-phase

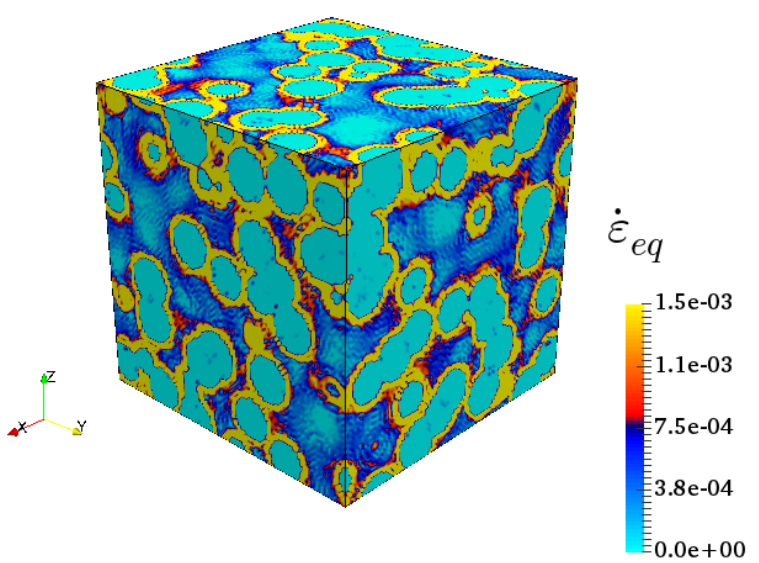

(b) $\simeq 50 \%$ of $\alpha$-phase

Figure 8: Distribution of the equivalent plastic strain rate within the austenitic phase during the transformation.

of Leblond et al. (1989)'s classical model of transformation plasticity. The new approach relies on a limit-analysis, based on a kinematical approach, of a model geometry consisting of a hollow sphere made of plastic $\gamma$-phase, subjected to two loadings, an external one due to the macroscopic applied stress, plus an internal one due to the volumetric transformation strain of an enclosed growing rigid nucleus of $\alpha$-phase (Figure 10).

The inner and outer radii of the hollow sphere are denoted $r_{f}$ and $R$ respectively, and are connected through the relation

$$
f=\frac{r_{f}^{3}}{R^{3}}
$$

where $f$ denotes the volume fraction of the daughter $\alpha$-phase like above. The whole domain, the outer spherical shell (mother-phase) and the inner core (daughter-phase) are denoted $\Omega, \Omega_{M}$ and $\Omega_{D}$ respectively. The outer and inner boundaries of $\Omega_{M}$, identical to $\partial \Omega$ and $\partial \Omega_{D}$, are subjected to conditions of homogeneous stress:

$$
\left\{\begin{array}{lllll}
\boldsymbol{\sigma}(\mathbf{x}) \cdot \mathbf{n}(\mathbf{x}) & =\boldsymbol{\Sigma} \cdot \mathbf{n}(\mathbf{x}) & \text { on } & \partial \Omega & (r=R) \\
\boldsymbol{\sigma}(\mathbf{x}) \cdot \mathbf{n}^{-}(\mathbf{x}) & =-\overline{\boldsymbol{\Sigma}} \cdot \mathbf{n}^{-}(\mathbf{x}) & \text { on } & \partial \Omega_{D} & \left(r=r_{f}\right)
\end{array}\right.
$$

where $\boldsymbol{\Sigma}$ represents the macroscopic applied stress and $\overline{\boldsymbol{\Sigma}}$ the internal loading stress. The components of the overall and internal strain tensors $(\mathbf{E}, \overline{\mathbf{E}})$ are defined as

$$
\left\{\begin{array}{l}
E_{i j}=\frac{1}{\operatorname{vol}(\Omega)} \int_{\partial \Omega} \frac{1}{2}\left(u_{i} n_{j}+u_{j} n_{i}\right) \mathrm{d} S \\
\bar{E}_{i j}=\frac{1}{\operatorname{vol}\left(\Omega_{D}\right)} \int_{\partial \Omega_{D}} \frac{1}{2}\left(u_{i} n_{j}^{+}+u_{j} n_{i}^{+}\right) \mathrm{d} S
\end{array}\right.
$$

where $\mathbf{u}$ denotes the displacement. The virtual power $\mathcal{P}_{e}$ of external forces then reads

$$
\mathcal{P}_{e}=\operatorname{vol}(\Omega) \Sigma_{i j} \dot{E}_{i j}+\operatorname{vol}\left(\Omega_{D}\right) \bar{\Sigma}_{i j} \dot{\bar{E}}_{i j}
$$

An analogy with problems of ductile rupture is exploited, through use of trial velocity fields similar to those considered 


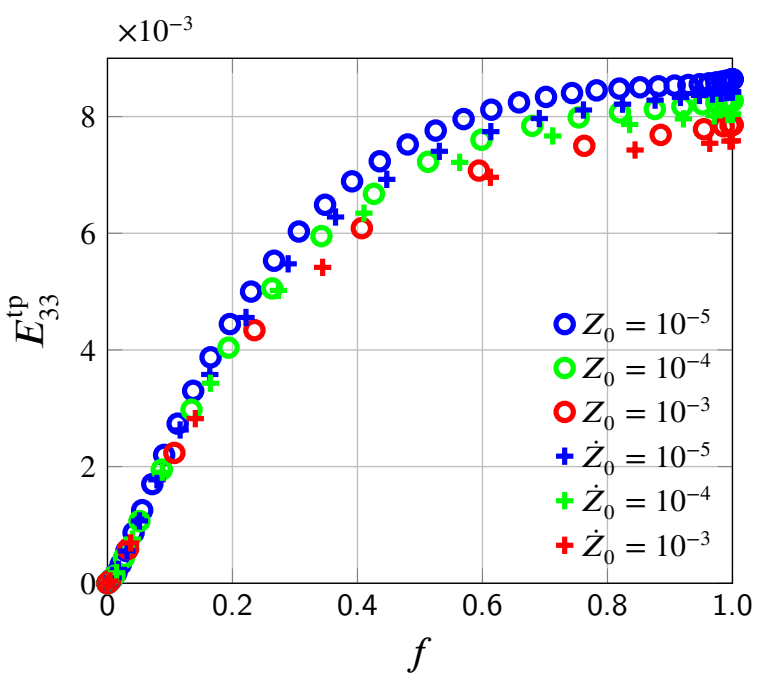

Figure 9: Comparison of transformation plastic strains with instantaneous (IN) and sporadic (SN) nucleation processes, for an applied stress of $80 \mathrm{MPa}$.

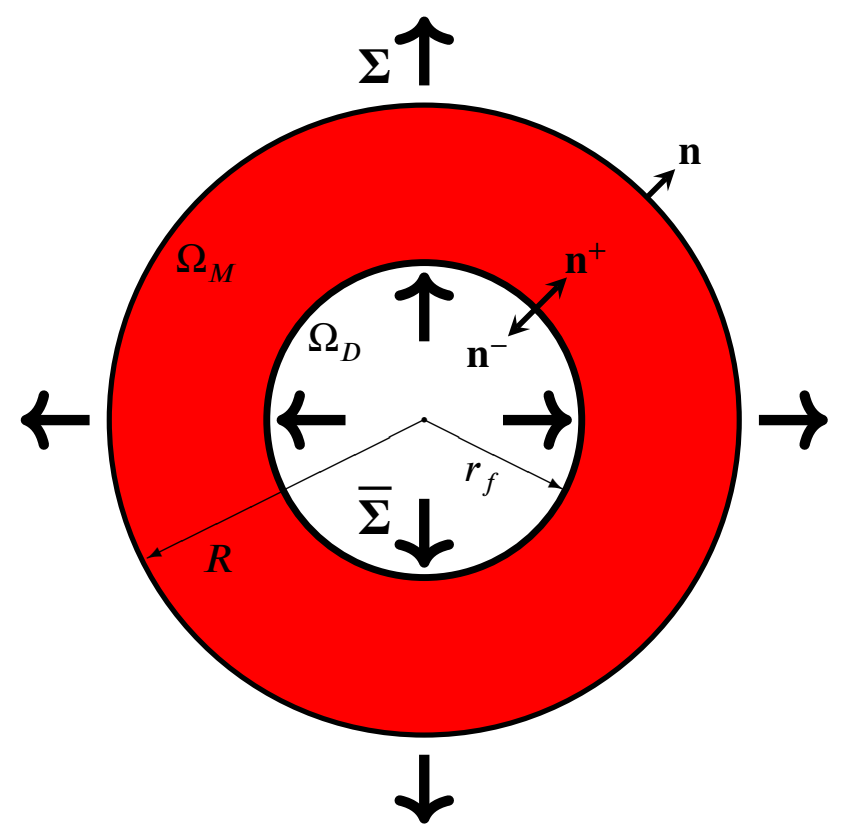

Figure 10: Microstructure considered for the limit-analysisbased approach of El Majaty et al. (2018).

in studies of hollow spheres with traction-free inner boundaries (no internal loading). However, the incompressible trial displacement fields needed are more numerous here; indeed they must account for all possible values of the 6 kinematics parameters $E_{1}, E_{2}, E_{3}$ and $\bar{E}_{1}, \bar{E}_{2} \bar{E}_{3}$ (eigenvalues of the tensors $\mathbf{E}$ and $\overline{\mathbf{E}}$ ) tied through the relation

$$
E_{1}+E_{2}+E_{3}=f\left(\bar{E}_{1}+\bar{E}_{2}+\bar{E}_{3}\right)
$$

resulting from incompressibility of the $\gamma$-phase. Five trial velocity fields are therefore used: three borrowed from the work of Gurson (1977) and two Eshelby-type fields proposed by Monchiet et al. (2011) to refine Gurson's analysis.

The calculation of the overall plastic dissipation is then the same as in the work of Monchiet et al. (2011); but the outputs, that is the overall yield criterion and the "double" overall plastic flow rule providing the values of both strain rates $\dot{\mathbf{E}}$ and $\dot{\overline{\mathbf{E}}}$, are different. The yield criterion reads:

$\Phi(\boldsymbol{\Sigma}, \overline{\boldsymbol{\Sigma}}, f)=\left(\frac{T_{e q}}{\sigma_{Y}^{M}}\right)^{2}+2 f \cosh \left(\frac{S_{H}}{\sigma_{Y}^{M}}\right)-1-f^{2}=0$,

where $\sigma_{Y}^{M}$ denotes the yield stress of the mother, $\gamma$-phase, and

$$
\left\{\begin{array}{l}
\mathbf{S}=\boldsymbol{\Sigma}+\overline{\boldsymbol{\Sigma}} \\
\mathbf{T}=\boldsymbol{\Sigma}+f \overline{\boldsymbol{\Sigma}} \\
S_{H}=\left[\frac{1}{4}(\operatorname{tr} \mathbf{S})^{2}+\frac{2}{3 g(f)} \mathbf{S}_{e q}^{2}\right]^{\frac{1}{2}} \\
g(f)=1-4 f \frac{\left(1-f^{2 / 3}\right)^{2}}{1-f}
\end{array}\right.
$$

Furthermore the double flow rule reads:

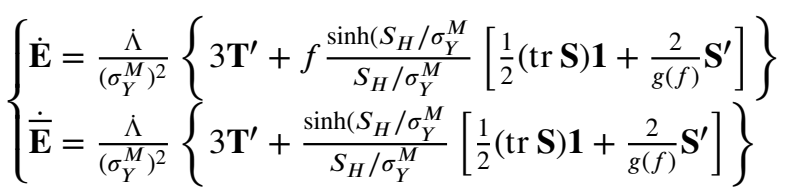

where a prime applied to a tensor denotes its deviatoric part, and $\dot{\Lambda}(\geq 0)$ is the overall plastic multiplier.

Application of this formalism to Greenwood-Johnson's mechanism (with the internal strain rate imposed by the growth of the nucleus of the rigid daughter $\alpha$-phase) leads to the following expression of the transformation plastic strain rate (El Majaty et al., 2018):

$$
\dot{\mathbf{E}}^{\mathrm{tp}}=\frac{4}{9} \frac{1}{\sigma_{Y}^{M}} \frac{\Delta V}{V} \frac{1-f}{f} \frac{1}{g(f)} \frac{\bar{X} / X-1}{Y} \boldsymbol{\Sigma}^{\prime} \dot{f},
$$

where the quantities

$$
X=\frac{\Sigma_{e q}}{\sigma_{Y}^{M}}, \quad \bar{X}=\frac{\bar{\Sigma}_{e q}}{\sigma_{Y}^{M}} \quad \text { and } \quad Y=\frac{1}{3} \frac{\operatorname{tr} \mathbf{S}}{\sigma_{Y}^{M}}
$$

may be calculated by combining the yield criterion and the flow rule.

This new approach brings major improvements to Leblond et al. (1989)'s classical model of transformation plasticity. It notably suppresses the need for ad hoc assumptions, and permits to consider more general loading situations, with overall applied stresses comparable in magnitude to the yield stress of the weaker $\gamma$-phase.

\section{Assessment of the model using FFT numerical simulations}

We now present comparisons of FFT numerical results and predictions of the limit-analysis theory. With this aim in 


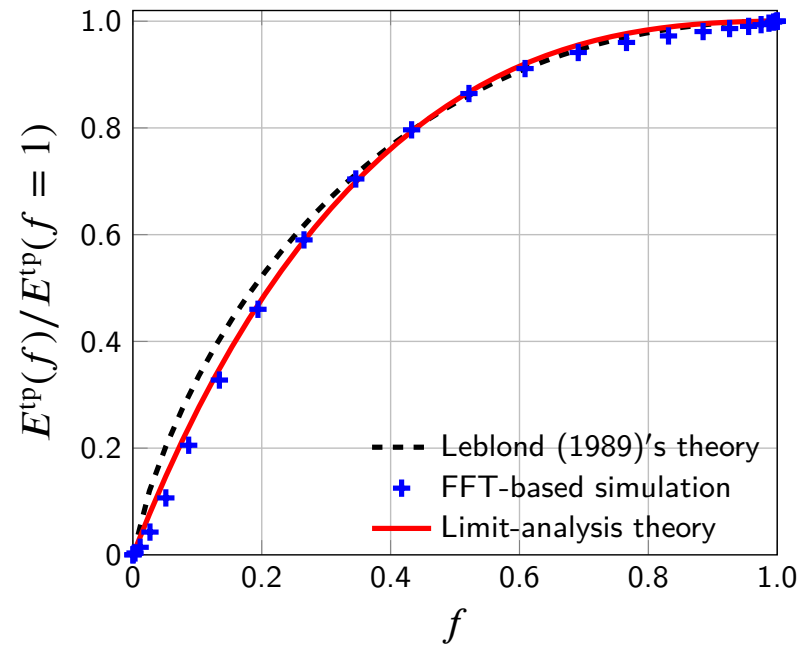

(a) Evolution of the normalized transformation plastic strain (tension)

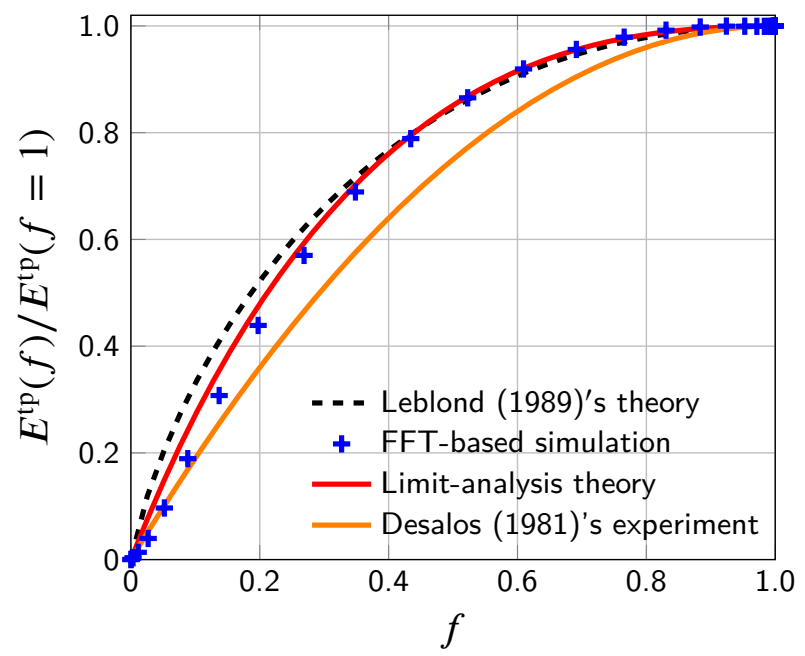

(c) Evolution of the normalized transformation plastic strain (compression)

Figure 11: Transformation plasticity for uniaxial loadings.

view, we consider: (i) the normalized transformation plastic strain, $E^{\mathrm{tp}}(f) / E^{\mathrm{tp}}(f=1)$, as a function of the volume fraction $f$, for an equivalent stress applied of $40 \mathrm{MPa}$; and (ii) the value of the transformation plastic strain after complete transformation, $E^{\operatorname{tp}}(f=1, \Sigma)$, as a function of the applied stress.

Figure 11 shows the results obtained for uniaxial loadings, for both tensile stresses (Figure 11a and Figure 11b) and compressive ones (Figure 11c and Figure 11d). The predictions of Leblond et al. (1989)'s original model are also displayed for reference; and Figure 11c and Figure 11d show in addition the old, but high-quality experimental results obtained by Desalos (1981) for compressive stresses not exceeding half of the yield stress of austenite, completed by

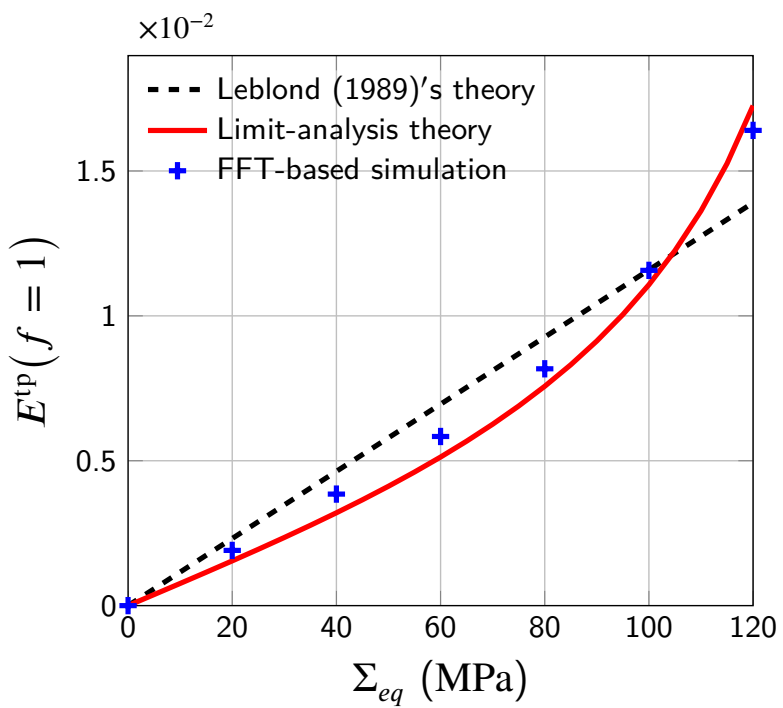

(b) Transformation plastic strain after full transformation (tension)

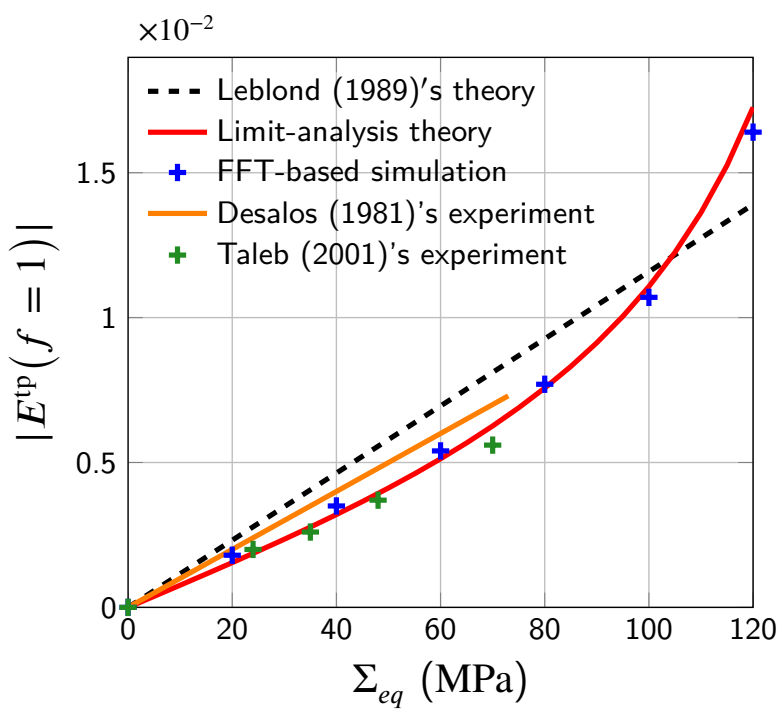

(d) Transformation plastic strain after full transformation (compression)

those of Taleb et al. (2001) for the transformation plastic strain after complete transformation.

Several points are noteworthy here:

- The predictions of El Majaty et al. (2018)'s new model of transformation plasticity are in excellent agreement with the results of the FFT simulations, for both the evolution of the normalized transformation plastic strain, and the value of the transformation plastic strain after complete transformation. The new model reproduces in particular the complex dependence, resulting from the simulations, of the transformation plastic strain after complete transformation upon the stress applied (linear for stresses less than about half of the yield stress of austenite, nonlinear above this value). 
- The results of the FFT simulations being taken as a reference, the predictions of Leblond et al. (1989)'s old theory are poorer than those of El Majaty et al. (2018)'s new one. For the evolution of the normalized transformation plastic strain, the old theory fails to predict a finite initial slope, as already noted in the Introduction. For the transformation plastic strain after complete transformation, it misses the nonlinear increase with the stress applied, for values of this stress exceeding half of the yield stress of austenite. Both of these points are captured by the new theory.

- The experimental results of Desalos (1981) for the evolution of the normalized transformation plastic strain agree slightly better with the predictions of El Majaty et al. (2018)'s new theory than with those of Leblond et al. (1989)'s old one. For the transformation plastic strain after complete transformation, the experimental results (for moderate stresses only) of Desalos (1981) do not permit to discriminate between the two theories, but those of Taleb et al. (2001) favour the new theory. (For large stresses comparable to the yield stress of austenite, no experiments have been performed for the material considered here; but the nonlinear increase of the transformation plastic strain after complete transformation with the stress applied is well-known experimentally for other materials, see for instance (Greenwood and Johnson, 1965)).

For further comparisons, we present the results of FFT simulations performed for multiaxial loadings. The form of the overall stress tensor applied corresponds to a combined tension/shear test:

$$
\boldsymbol{\Sigma}=\left(\begin{array}{ccc}
\Sigma & \mathcal{T} & 0 \\
\mathcal{T} & 0 & 0 \\
0 & 0 & 0
\end{array}\right)
$$

Various values of the ratio $\eta=\Sigma / \mathcal{T}$ are considered, $\eta=$ $0.5,1,2$. FFT results are reported in Figure 12 for the strain components $E_{11}^{\mathrm{tp}}$ and $E_{12}^{\mathrm{tp}}$, together with the predictions of El Majaty et al. (2018)'s new theory. An almost perfect agreement is again obtained for all values of the $\eta$ ratio in the whole stress range.

\section{Conclusion}

In this article, we provided new results on transformation plasticity induced by (Greenwood and Johnson, 1965)'s mechanism, by making use of an FFT-based numerical scheme. Elementary cells with a number of $\alpha$-phase nuclei growing spherically were considered, in order to take into account the effect of mechanical interactions between nuclei.

It was found that instantaneous and sporadic nucleation processes lead to basically similar results for the overall transformation plastic strain.

The numerical computations were used to assess, upon consideration of realistic microstructures, a recently proposed limit-analysis-based theory of transformation plasticity (El Majaty et al., 2018). It was shown that this new theory leads to accurate estimates of the transformation plasticity phenomenon over a wide range of applied stresses, for both uniaxial and multiaxial loadings. In particular, the nonlinear increase of the transformation plastic strain after complete transformation with the stress applied is very well predicted.

This work paves the way for further numerical studies of transformation plasticity, using Moulinec and Suquet (1998)'s powerful FFT-based numerical scheme. One point of special interest would be the effect of the possible spheroidal growth of $\alpha$-phase nuclei, which may happen in austenitic (anisotropic) microstructures exhibiting segregation bands (Desalos, 1981). This type of growth is known to destroy the overall geometric isotropy of the material, and thus lead to interesting new effects, such as the non-vanishing of the transformation plastic strain in the absence of any external stress (Desalos, 1981). This feature will be investigated in a future work.

\section{A. Effect of homogeneous/heterogeneous nucleation}

Some observations of the microstructure during the $\gamma \rightarrow$ $\alpha$ transformation have shown that most of the nuclei appear at grain boundaries (Offerman et al., 2006). This phenomenon is named heterogeneous nucleation, in contrast to homogeneous nucleation for which nuclei appear totally randomly in space (stochastic events).

A natural consequence of this new type of nucleation is the increase of the continuum percolation threshold $p_{c}$, defined as the critical value of the fraction of $\alpha$-phase giving rise to a percolating cluster (there exists a continuous path between opposite faces of the cell, lying entirely within the $\alpha$-phase). The reason is that for heterogeneous nucleation, the centres of nuclei are more spaced on average than for homogeneous nucleation, so that the growing nuclei remain separated from each other for a longer time; see Figure 13 which shows three different microstructures at the percolation threshold.

To simulate heterogeneous nucleation, some authors modelled the austenite microstructure with a Voronoï tesselation, and then took the vertices as nucleation sites (Quey et al., 2011). The method employed here, which permits an easier control of the percolation threshold, is different. We first use a random sequential adsorption (RSA) algorithm to generate a microstructure with monodisperse or polydisperse nonoverlapping nuclei, with a volume fraction of $35 \%$. Then we take the centre of each nucleus as an instantaneous or latent site of nucleation activation.

New FFT simulations are then performed for instantaneous heterogeneous nucleation, considering the same values of the instantaneous nucleation density as for homogeneous nucleation, $Z_{0}=10^{-5}, 10^{-4}, 10^{-3}$. The percolation threshold $p_{c}$ is almost independent of the value of $Z_{0}$ but is larger than for homogeneous nucleation $\left(p_{c}=0.35\right.$ instead of 0.29). 


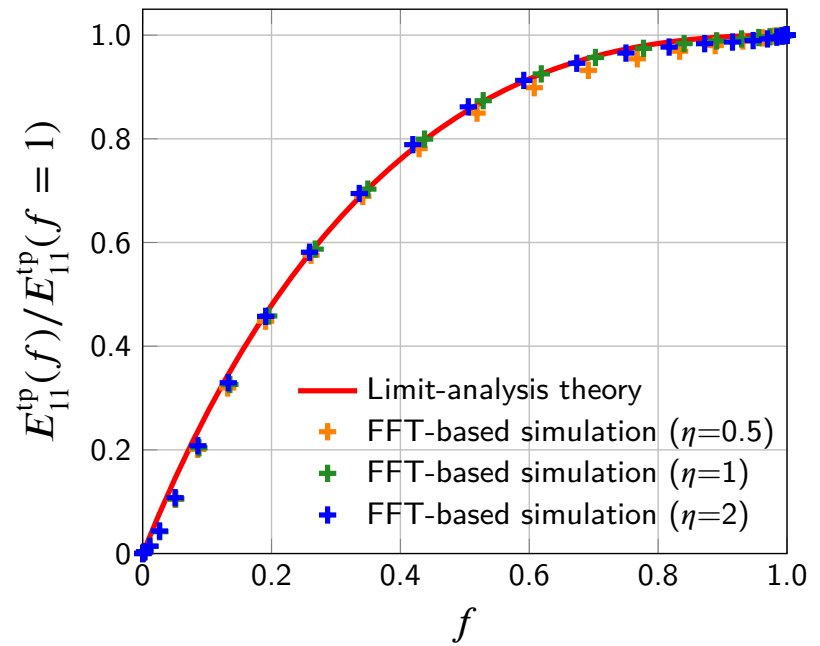

(a) Evolution of the normalized transformation plastic strain $E_{11}^{\mathrm{tp}}$

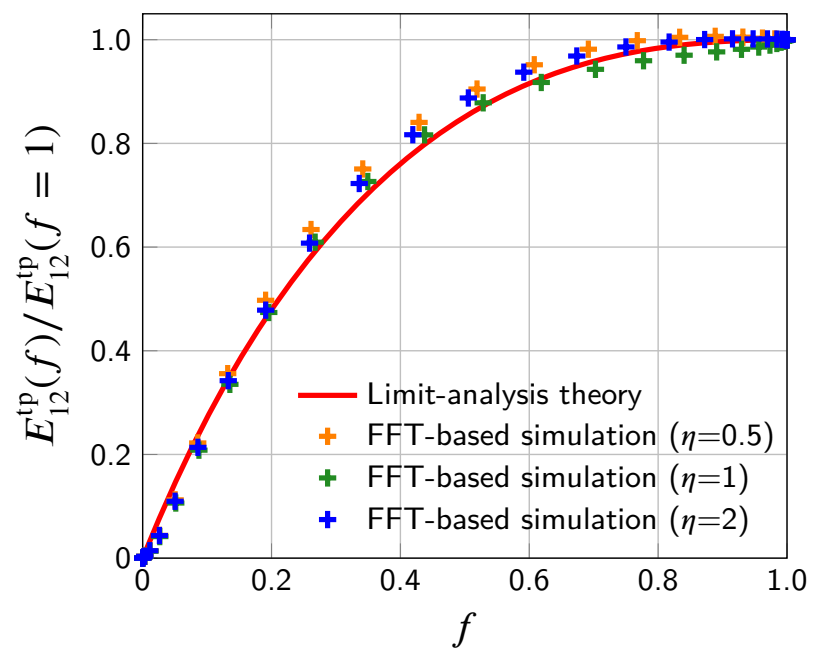

(c) Evolution of the normalized transformation plastic strain $E_{12}^{\mathrm{tp}}$

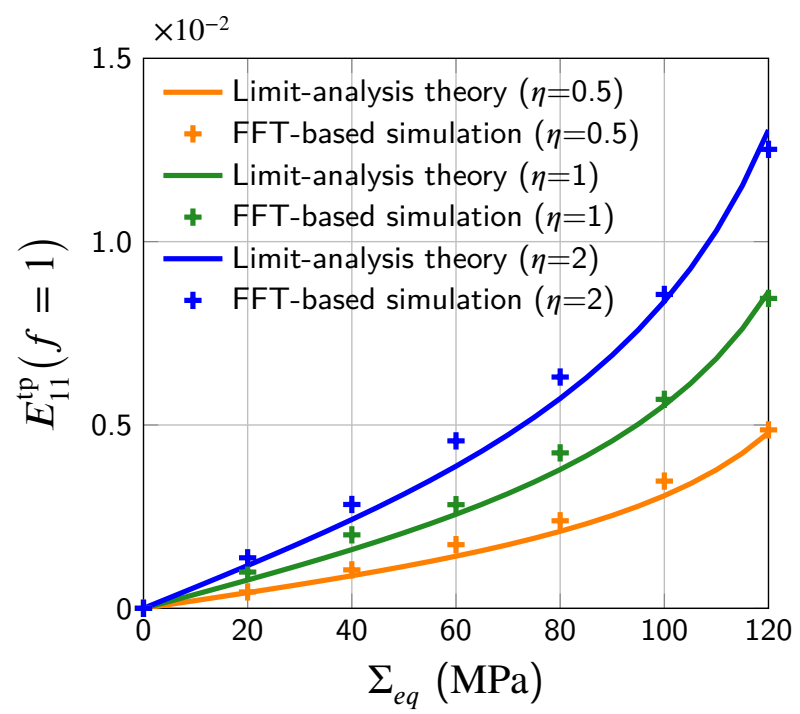

(b) Transformation plastic strain $E_{11}^{\text {tp }}$ after full transformation

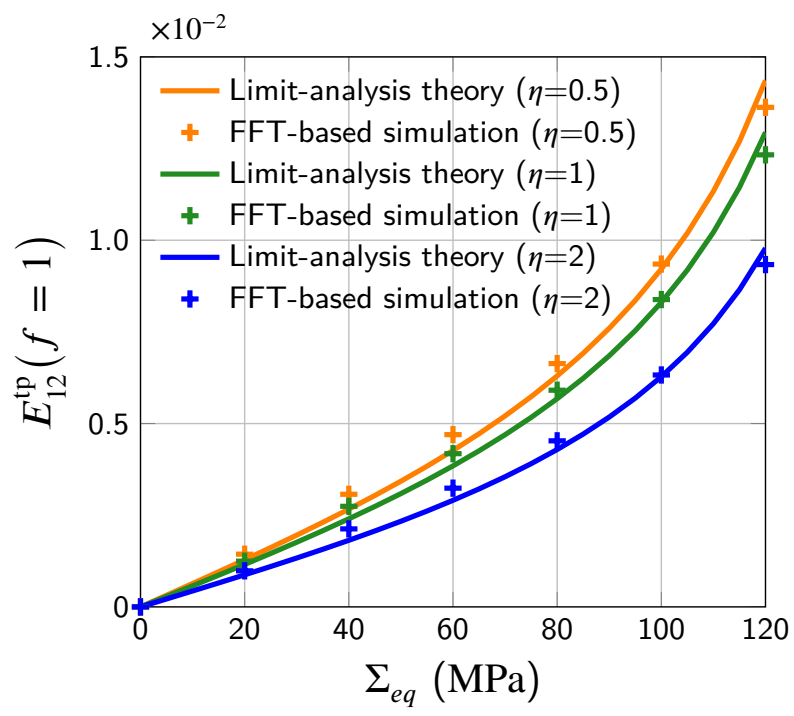

(d) Transformation plastic strain $E_{12}^{\mathrm{tp}}$ after full transformation

Figure 12: Transformation plasticity for tension-shear tests, with different values of the ratio $\eta=\Sigma / \mathcal{T}$.
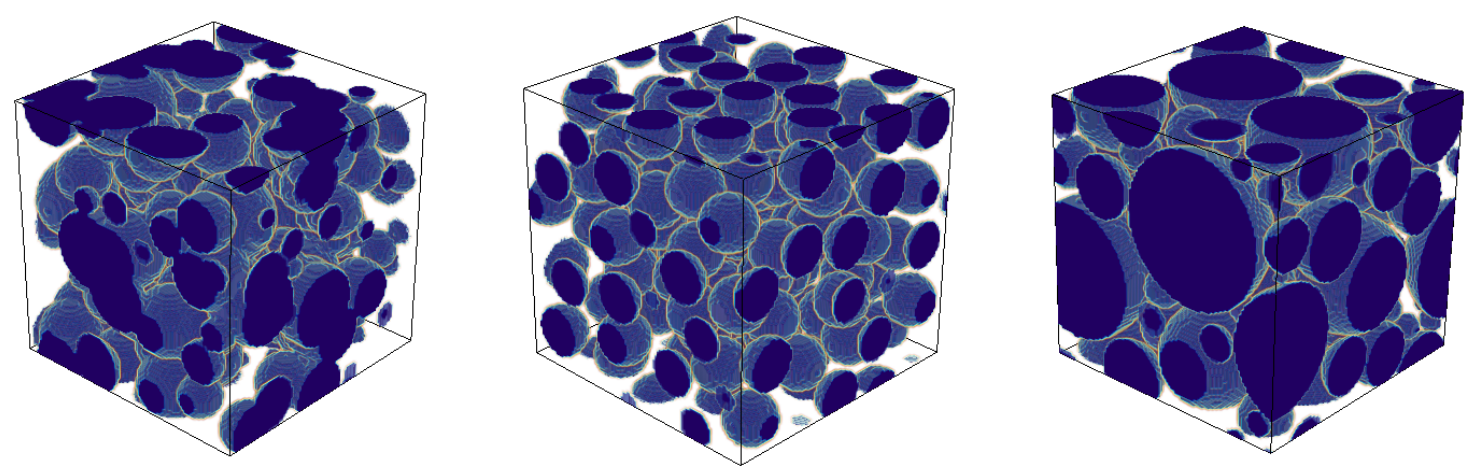

Figure 13: Various isotropic microstructures at the percolation threshold: (left) sporadic homogeneous nucleation, $p_{c} \simeq 0.29$; (centre) instantaneous heterogeneous nucleation, $p_{c} \simeq 35 \%$; (right) sporadic heterogeneous nucleation $p_{c} \simeq 60 \%$. 


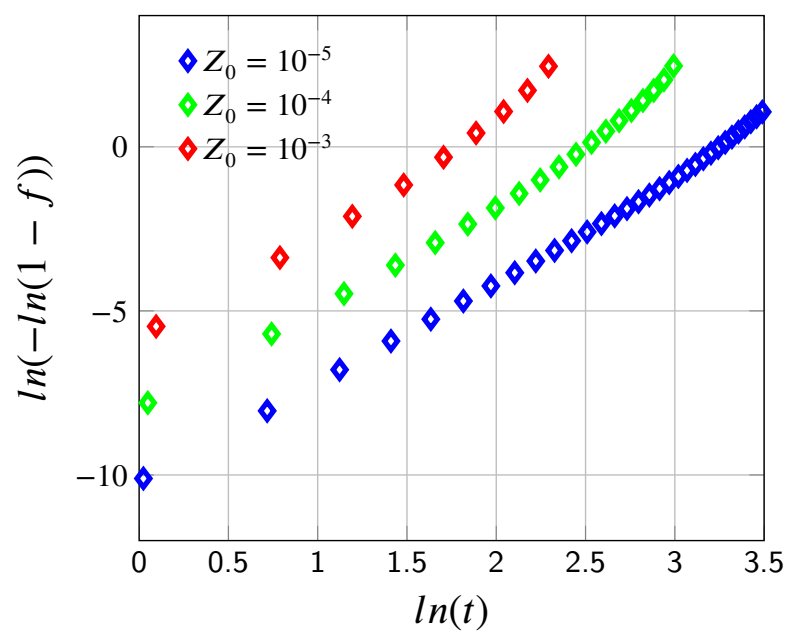

(a) Fit of numerical coefficients of the JMAK model

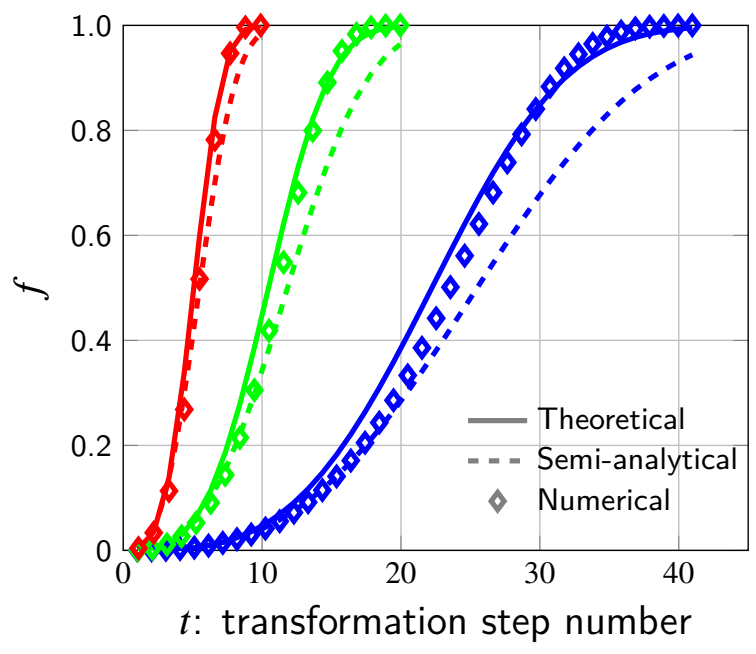

(b) Extent of transformation

Figure 14: Comparison between the JMAK model and instantaneous heterogeneous nucleation simulations based on a RSA algorithm, for three values of the instantaneous density of nucleation $Z_{0}$.

Figure 14 shows the results obtained for the transformation kinetics, as Figure 3 and Figure 4 did for homogeneous nucleation. One can observe that the kinetics no longer perfectly follow a JMAK-type model; this is certainly because the derivation of this model was based on the hypothesis of homogeneous nucleation, which is no longer respected here.

Figure 15a compares the results obtained for the transformation plastic strain, for instantaneous homogeneous and heterogeneous nucleation. One sees that the transformation plastic strain is slightly larger for heterogeneous nucleation than for homogeneous nucleation. This can be explained through the correlation which exists between the homogeneous or heterogeneous character of nucleation and the value of the "specific area" $S_{A}$ of the interfacial surface, defined as the total number of voxels undergoing the transformation between two successive transformation steps, divided by the total number of voxels in the cell. Figure $15 \mathrm{~b}$ illustrates this correlation: the specific area is systematically larger for heterogeneous nucleation than for homogeneous nucleation. Since transformation plasticity, in Greenwood and Johnson (1965)'s mechanism, is due to the plastic accommodation of the mother phase which surrounds the daughter phase, the larger the area of the interfacial surface, the larger this mechanism must be; thus it is only to be expected that heterogeneous nucleation generate a larger transformation plastic strain.

\section{B. Influence of some parameters of the numerical representation of the transformation}

\section{B.1. Yield stress variation during the transformation}

It was explained in subsubsection 4.2.4 that each timeinterval $[t, t+\Delta t]$ corresponding to a given transformation step is divided into 5 substeps, the function $\zeta(\mathbf{x})$ taking the successive values $0,0.2,0.4,0.6,0.8,1$ at each voxel $\mathbf{x}$ undergoing the transformation over this time-interval. It was also mentioned that the yield stress $\sigma_{Y}(\mathbf{x})$ at such a voxel is considered to vary from the value $\sigma_{Y}^{\gamma}$ for the $\gamma$-phase to that, $\sigma_{Y}^{\alpha}$ for the $\alpha$-phase, over the interval $\zeta(\mathbf{x}) \in[0.8,1]$, that is at the end of the transformation rather than at its beginning or during the entire transformation.

The physical reason for doing so was explained above. Nevertheless, one may study the effect of the position of the interval of $\zeta(\mathbf{x})$-values over which $\sigma_{Y}(\mathbf{x})$ is considered to vary from $\sigma_{Y}^{\gamma}$ to $\sigma_{Y}^{\alpha}$. We thus decided to vary $\sigma_{Y}(\mathbf{x})$ over various intervals $\left[\zeta_{Y}, \zeta_{Y}+0.2\right]$, with $\zeta_{Y}$ taking the values 0 , $0.2,0.4,0.6,0.8$.

Figure 16 shows the evolution of the transformation plastic strain for the various values of $\zeta_{Y}$ (for the same, single realization of the microstructure). The transformation plastic strain is somewhat smaller when the value of $\zeta_{Y}$ is low; this is natural since the zone transformed becomes harder earlier then.

\section{B.2. Thickness of the transforming layer}

It was mentioned in subsubsection 4.2.3 that each spherical nucleus of $\alpha$-phase is considered to increase by one voxel in the radial direction at each transformation step; this defines a "radial growth rate" $G=1 / v$ in an elementary cell containing $v^{3}$ voxels (see subsubsection 4.2.3).

One may investigate the effect of the number of voxels added in the radial direction to each nucleus at each transformation step. This is equivalent to examining the influence of the thickness of the transforming layer, or in other terms that of the number of transformation steps used to discretize the full transformation of the cell. We did so by performing three simulations with 1, 2 and 3 voxels added in the radial direction per transformation step, corresponding to radial growth 


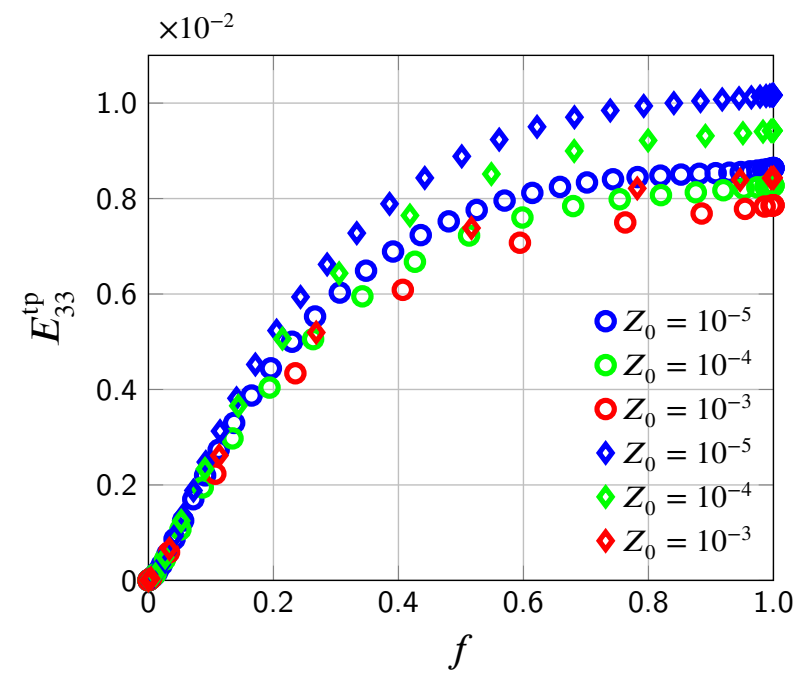

(a) Transformation plastic strain vs volume fraction of $\alpha$-phase

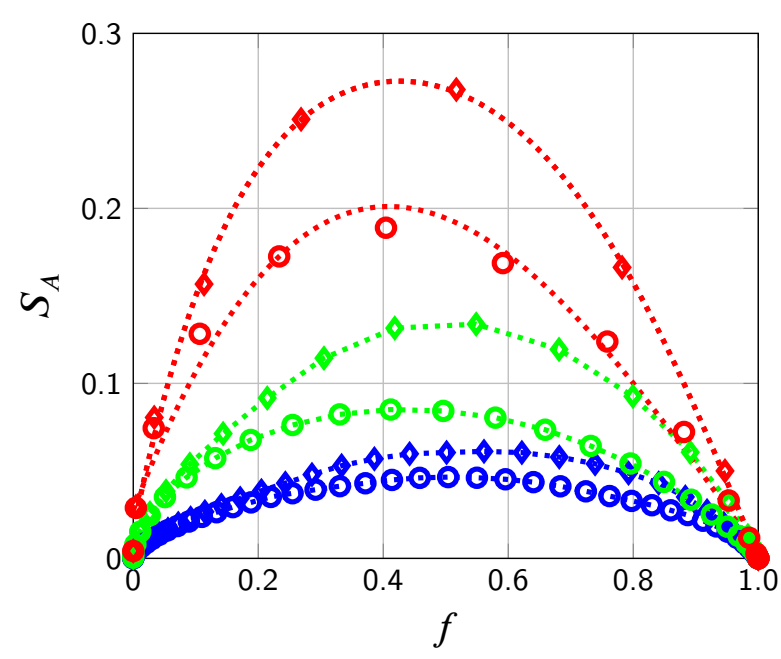

(b) Specific surface area $S_{A}$ vs volume fraction of $\alpha$-phase

Figure 15: Comparison between instantaneous heterogeneous nucleation (symbols $\diamond$ ) and instantaneous homogeneous nucleation (symbols o), for an applied stress of $80 \mathrm{MPa}$.

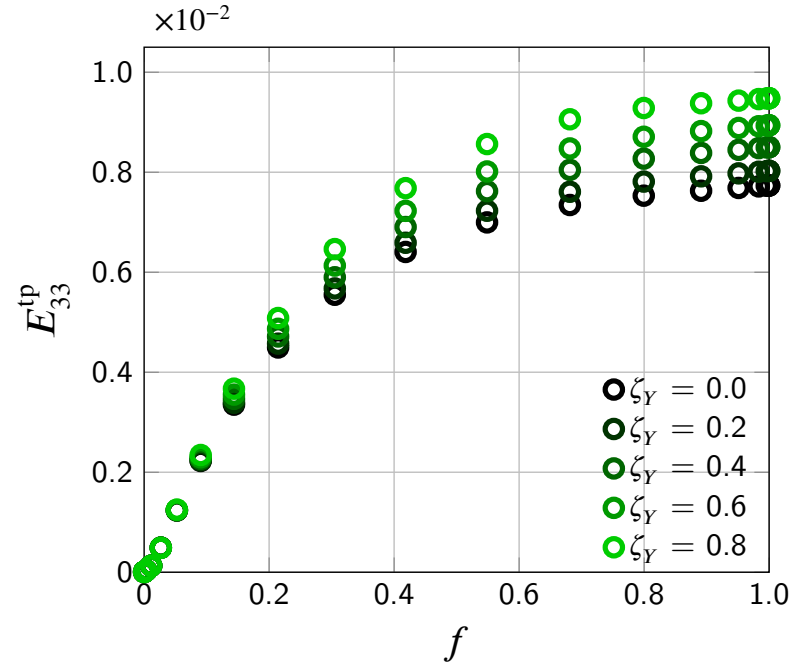

Figure 16: Comparison of transformation plastic strains with different evolutions of the yield stress during the transformation, for an applied stress of $80 \mathrm{MPa}$. Instantaneous heterogeneous nucleation.

rates $G=1 / v, 2 / v, 3 / v$, in a cell containing $v^{3}=100^{3}$ voxels.

Figure 17 shows the results obtained for the transformation kinetics. Apart from the trivial quicker evolution of the fraction $f$ of daughter-phase resulting from the increase of $G$, these results show little qualitative influence of this parameter.

Figure 18 illustrates the results obtained for transformation plasticity (for the same, single realization of the microstructure). Again, the influence of the value of the radial growth $G$ is quite small.

Figure 17 and Figure 18 thus lead to the conclusion that the number of transformation steps corresponding to the value
$G=1 / v$ is sufficient to get converged, meaningful results for both the transformation kinetics and transformation plasticity.

\section{References}

Barbe, F., Quey, R., 2011. A numerical modelling of 3D polycrystal-topolycrystal diffusive phase transformations involving crystal plasticity. Int. J. Plast. 27, 823-840.

Barbe, F., Quey, R., Taleb, L., 2007. Numerical modelling of the plasticity induced during diffusive transformation. Case of a cubic array of nuclei. Eur. J. Mech. A. Solids 26, 611-625.

Barbe, F., Quey, R., Taleb, L., de Cursi, E.S., 2008. Numerical modelling of the plasticity induced during diffusive transformation. An ensemble averaging approach for the case of random arrays of nuclei. Eur. J. Mech. A. Solids 27, 1121-1139.

Brenner, R., Lebensohn, R., Castelnau, O., 2009. Elastic anisotropy and yield surface estimates of polycrystals. Int. J. Solids Struct. 46, 30183026.

Cherkaoui, M., Berveiller, M., Lemoine, X., 2000. Couplings between plasticity and martensitic phase transformation: overall behavior of polycrystalline TRIP steels. Int. J. Plast. 16, 1215-1241.

Coret, M., Calloch, S., Combescure, A., 2002. Experimental study of the phase transformation plasticity of 16MND5 low carbon steel under multiaxial loading. Int. J. Plast. 18, 1707-1727.

Coret, M., Calloch, S., Combescure, A., 2004. Experimental study of the phase transformation plasticity of 16MND5 low carbon steel induced by proportional and nonproportional biaxial loading paths. Eur. J. Mech. A Solids 23, 823-842.

Desalos, Y., 1981. Comportement mécanique et dilatométrique de l'austénite métastable de l'acier A533. IRSID report 95349401.

Diani, J., Sabar, H., Berveiller, M., 1995. Micromechanical modelling of the transformation induced plasticity (TRIP) phenomenon in steels. Int. J. Eng. Sci. 33, 1921-1934.

El Majaty, Y., Leblond, J.B., Kondo, D., 2018. A novel treatment of Greenwood-Johnson's mechanism of transformation plasticity - Case of spherical growth of nuclei of daughter-phase. J. Mech. Phys. Solids 121, 175-197.

Fischlschweiger, M., Cailletaud, G., Antretter, T., 2012. A mean-field model for transformation induced plasticity including backstress effects for non-proportional loadings. Int. J. Plast. 37, 53-71.

Fukumuto, M., Yoshizaki, M., Imataka, H., Okamua, K., Yamamoto, K., 
FFT-based simulations of transformation plasticity

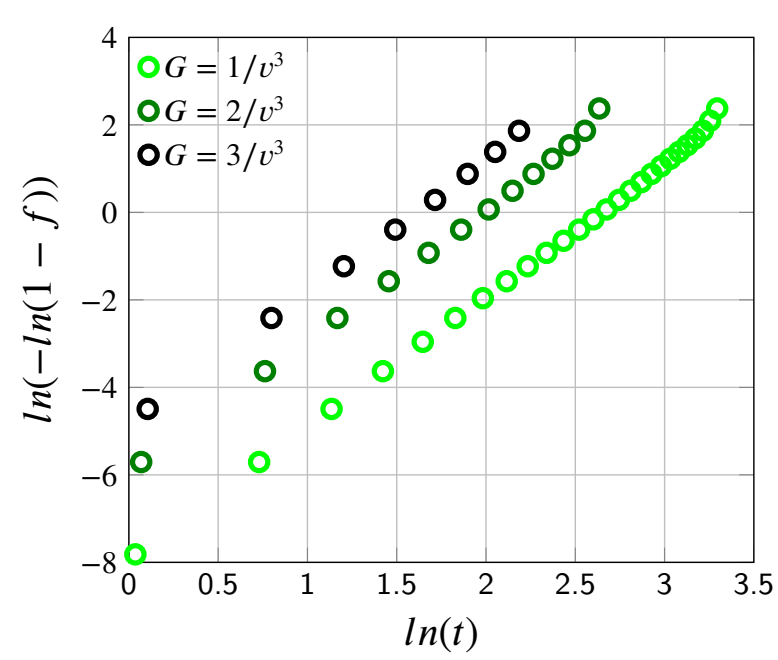

(a) Fit of numerical coefficients of the JMAK model

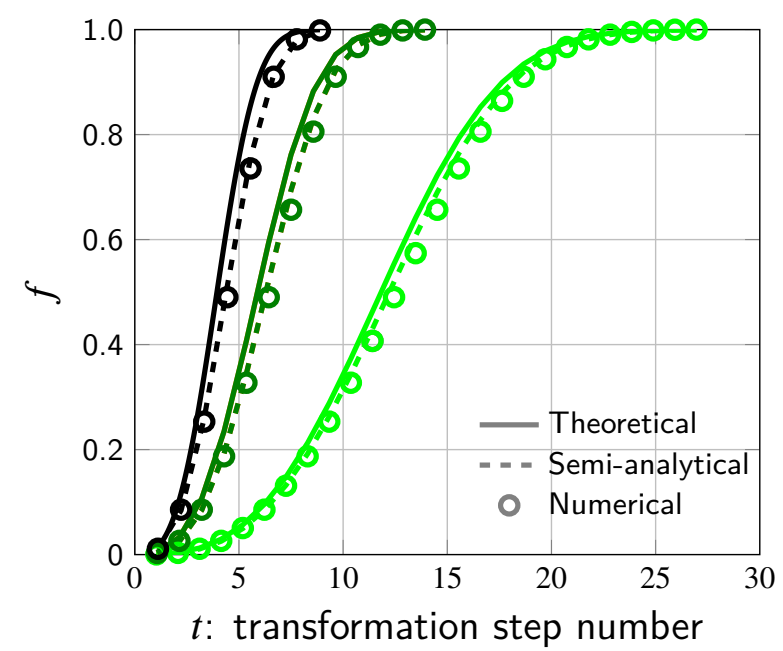

(b) Extent of transformation

Figure 17: Comparison between the JMAK model and instantaneous homogeneous nucleation simulations, for different radial growth rates $G$.

2001. Three-dimensional FEM analysis of helical gear subjected to the carburized quenching process. J. Soc. Mater. Sci. Japan 50, 598-605.

Ganghoffer, J., Denis, S., Gautier, E., Simon, A., Sjostrom, S., 1992. Finite element calculation of the micromechanics of a diffusional transformation, in: Mechanical Behaviour of Materials VI. Elsevier, pp. 165-170.

Greenwood, G.W., Johnson, R., 1965. The deformation of metals under small stresses during phase transformations. Proc. R. Soc. London A. 283, 403-422.

Gurson, A.L., 1977. Continuum theory of ductile rupture by void nucleation and growth: Part I Yield criteria and flow rules for porous ductile media. J. Eng. Mater. Technol. 99, 2-15.

Hill, R., 1967. The essential structure of constitutive laws for metal composites and polycrystals. J. Mech. Phys. Solids 15, 79-95.

Kempen, A., Sommer, F., Mittemeijer, E., 2002. Determination and interpretation of isothermal and non-isothermal transformation kinetics; the effective activation energies in terms of nucleation and growth. J. Mater. Sci. 37, 1321-1332.

Lebensohn, R.A., Brenner, R., Castelnau, O., Rollett, A.D., 2008. Orientation image-based micromechanical modelling of subgrain texture evolution in polycrystalline copper. Acta Mater. 56, 3914-3926.

Lebensohn, R.A., Kanjarla, A.K., Eisenlohr, P., 2012. An elasto-

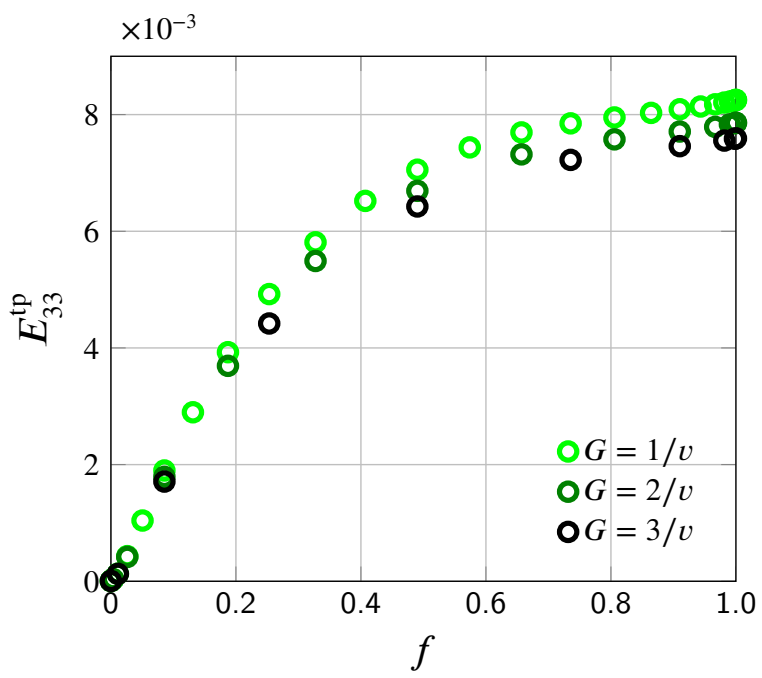

Figure 18: Comparison of transformation plastic strains for different radial growth rates $G$, for an applied stress of 80 $\mathrm{MPa}$. Instantaneous homogeneous nucleation.

viscoplastic formulation based on fast Fourier transforms for the prediction of micromechanical fields in polycrystalline materials. Int. J. Plast. 32, 59-69.

Leblond, J.B., Devaux, J., Devaux, J., 1989. Mathematical modelling of transformation plasticity in steels I: case of ideal-plastic phases. Int. J. Plast. 5, 551-572.

Lee, S.B., Lebensohn, R., Rollett, A.D., 2011. Modeling the viscoplastic micromechanical response of two-phase materials using Fast Fourier Transforms. Int. J. Plast. 27, 707-727.

Magee, C.L., Paxton, H.W., 1966. Transformation kinetics, microplasticity and aging of martensite in Fe-31 Ni. Technical Report. Carnegie Inst. of Tech., Pittsburgh, PA.

Mandel, J., 1966. Contribution théorique à l'étude de l'écrouissage et des lois de l'écoulement plastique, in: Applied Mechanics. Springer, pp. 502-509.

Miyao, K., Wang, Z., Inoue, T., 1986. Analysis of temperature, stress and metallic structure in carburized-quenched gear considering transformation plasticity. J. Soc. Mater. Sci. Japan 35, 1352-1357.

Monchiet, V., Charkaluk, E., Kondo, D., 2011. A micromechanics-based modification of the Gurson criterion by using Eshelby-like velocity fields. Eur. J. Mech. A. Solids 30, 940-949.

Moulinec, H., Suquet, P., 1998. A numerical method for computing the overall response of nonlinear composites with complex microstructure. Comput. Meth. Appl. Mech. Eng. 157, 69-94.

Offerman, S., Van Dijk, N., Sietsma, J., Lauridsen, E., Margulies, L., Grigull, S., Poulsen, H., van der Zwaag, S., 2006. Phase transformations in steel studied by 3DXRD microscopy. Nucl. Instrum. Meth. Phys. Res. B 246, 194-200.

Offerman, S., Van Wilderen, L., Van Dijk, N., Sietsma, J., Rekveldt, M.T., Van der Zwaag, S., 2003. In-situ study of pearlite nucleation and growth during isothermal austenite decomposition in nearly eutectoid steel. Acta Mater. 51, 3927-3938.

Otsuka, T., 2014. Modélisation micromécanique de la plasticité de transformation dans les aciers par homogénéisation numérique fondée sur la TFR. Ph.D. thesis. Université Paris 13.

Otsuka, T., Brenner, R., Bacroix, B., 2018. FFT-based modelling of transformation plasticity in polycrystalline materials during diffusive phase transformation. Int. J. Eng. Sci. 127, 92-113.

Quey, R., Dawson, P., Barbe, F., 2011. Large-scale 3D random polycrystals for the finite element method: Generation, meshing and remeshing. Comput. Meth. Appl. Mech. Eng. 200, 1729-1745.

Suquet, P., Moulinec, H., Castelnau, O., Montagnat, M., Lahellec, N., Grennerat, F., Duval, P., Brenner, R., 2012. Multi-scale modeling of the me- 
chanical behavior of polycrystalline ice under transient creep. Procedia IUTAM 3, 76-90.

Taleb, L., Cavallo, N., Waeckel, F., 2001. Experimental analysis of transformation plasticity. Int. J. Plast. 17, 1-20.

Taleb, L., Petit, S., Jullien, J.F., 2004. Prediction of residual stresses in the heat affected zone, in: J. Phys. IV (Proceedings), EDP sciences. pp. 705-712.

Taleb, L., Sidoroff, F., 2003. A micromechanical modeling of the Greenwood-Johnson mechanism in transformation induced plasticity. Int. J. Plast. 19, 1821-1842.

Vincent, Y., Bergheau, J.M., Leblond, J.B., 2003. Viscoplastic behaviour of steels during phase transformations. Comptes-Rendus Mécanique 331, 587-594.

Weisz-Patrault, D., 2017. Multiphase model for transformation induced plasticity. Extended Leblond's model. J. Mech. Phys. Solids 106, 152175.

Wong, S.L., Madivala, M., Prahl, U., Roters, F., Raabe, D., 2016. A crystal plasticity model for twinning- and transformation-induced plasticity. Acta Mater. 118, 140-151. 